\title{
Modeling and Reduction of SAR Interferometric Phase Noise in the Wavelet Domain
}

\author{
Carlos López-Martínez and Xavier Fàbregas
}

\begin{abstract}
This paper addresses the problem of interferometric phase noise reduction in synthetic aperture radar interferometry. A new phase noise model in the complex domain is introduced and validated by using both simulated and real interferograms. This noise model is also derived in the complex wavelet domain, where a novel noise reduction algorithm, which is not based on a windowing process and without the necessity of phase unwrapping, is addressed. The use of the wavelet transform allows to maintain the spatial resolution in the filtered phase image and prevents to filter low coherence areas. By using both, simulated as well as real interferometric phase images, the performance of this algorithm, in terms of noise reduction, spatial resolution maintenance, and computational efficiency, is reported and compared with other conventional filtering approaches.
\end{abstract}

Index Terms-Phase noise modeling, phase noise reduction, SAR interferometry, wavelet transform.

\section{INTRODUCTION}

$\mathbf{S}$ AR INTERFEROMETRY (InSAR) is an established technique for the extraction of height information by means of SAR remote sensing [1], [2]. The height map generation process is divided in two main steps. First, an interferogram is generated using a pair of high-resolution complex SAR images of the same scene, but taken from slightly different locations. The images can be taken in the same survey (single-pass interferometry) or in different surveys (repeat-pass interferometry) over the scene. Afterward, the phase difference between both SAR images is transformed to height information. InSAR has been successfully applied with different sensors: spaceborne, i.e., the European Remote Sensing satellites ERS-1 and ERS-2 and Spaceborne Imaging Radar version C (SIR-C)/Spaceborne Imaging Radar-C/X-band Synthetic Aperture Radar (X-SAR) missions, and airborne, i.e., the German E-SAR system. InSAR data have a wide range of applications as, for instance, digital elevation model (DEM) generation, surface motion estimation [3], or vegetation structure retrieval [4] making use also of polarimetric SAR information.

The statistical coherence between both SAR images gives information about SAR images similarity and about the interferometric phase noise content, in such a way that any effect re-

Manuscript received December 17, 2001; revised July 8, 2002. This work was supported by EC-TMR Polarimetry Network ERB-RMRX-CT98-0211 and the Spanish CICYT project TIC1999-1050-C03-01. The authors thank the Generalitat de Catalunya for its financial support.

C. López-Martínez is with the German Aerospace Center (DLR), D-82234, Wessling, Germany.

X. Fàbregas is with the Technical University of Catalonia (UPC), E-08034, Barcelona, Spain.

Digital Object Identifier 10.1109/TGRS.2002.806997 ducing coherence will increase phase noise. Coherence reduction has different sources: due to the SAR system (system noise or geometric decorrelation for instance) or due to the scatterer (temporal decorrelation or volume decorrelation). The interferometric phase is wrapped within the interval $[-\pi, \pi)$, making necessary an unwrapping process to estimate the absolute phase [5]. The phase noise hinders this unwrapping process, as it is one of the causes of the phase residues [6], but it also affects the quality of the obtained DEM.

Interferometric phase statistics, under the assumption of a sufficiently large number of scatterers inside the resolution cell, have been completely characterized [7], [8]. It is also known that the phase noise, in the real domain, follows an additive noise model [9]. In this paper, a new complex interferometric phase noise model, both in the original and in the wavelet domain, is presented.

Several techniques have been proposed in the literature to reduce interferometric phase noise. The first approach, applied to the complex interferometric signal, is the multilook filter [10], reducing noise at the expense of spatial resolution. In [9], a technique applied to the real, as well as to the complex interferometric phase signals, and based on the local statistics filter [11], was presented. Furthermore, techniques reducing phase noise in the Fourier domain have been proposed [12]. The last two filters reduce noise and maintain, to a certain degree, the original spatial resolution. All these filters are based on a windowing process of the original signal in order to adapt either, to the signal or to the noise nonstationarities, or also to both of them. In [9], the windowing process is more complex, as it adapts to the signal morphology, but using a reduced set of windows. Consequently, the filter performance depends highly on the window dimensions. In this paper, and based on the developed complex phase noise model, a novel noise reduction technique in the wavelet domain is presented.

In Section II, the interferometric phase noise model in the complex domain is derived. The validity of the model is demonstrated using simulated as well as experimental interferograms. In Section III, a new phase noise filter in the wavelet domain, based on the developed noise model, is proposed. Section IV presents the performance of the algorithm by employing simulated and real interferograms and compares it with alternative algorithms. The conclusions are drawn in Section V.

\section{Complex Phase NoISE Model}

Interferometric phase is due to the interaction of two complex SAR images. The statistical behavior of the interferometric 
phase noise depends on this interaction. The interferometric phase quality is controlled by the amplitude of the correlation coefficient

$$
\rho=\frac{E\left\{I_{1} I_{2}^{*}\right\}}{\sqrt{E\left\{\left|I_{1}^{2}\right|\right\} E\left\{\left|I_{2}^{2}\right|\right\}}}=|\rho| e^{j \theta}
$$

where $I_{1}$ and $I_{2}$ are complex SAR images; $|\rho|$ is the coherence; and $\theta$ is the corresponding interferometric phase.

The interferometric phase has been completely characterized in the real domain. Several authors have independently derived, based on a Gaussian scattering model for distributed scatterers, the expression of the multilook interferometric phase probability density function (pdf) [7], [8]

$$
\begin{aligned}
& p_{\phi}(\phi)=\frac{\Gamma\left(n+\frac{1}{2}\right)\left(1-|\rho|^{2}\right)^{n} \beta}{2 \sqrt{\pi} \Gamma(n)\left(1-\beta^{2}\right)^{n+1 / 2}} \\
&+\frac{\left(1-|\rho|^{2}\right)^{n}}{2 \pi} F\left(n, 1 ; \frac{1}{2} ; \beta^{2}\right)
\end{aligned}
$$

where $\beta=|\rho| \cos (\phi-\theta) ; F$ represents the Gauss hypergeometric function; and $n$ is the number of looks. Equation (2) is symmetric $(\bmod 2 \pi)$ about its mode, which occurs at $\theta$. This pdf has been proved to be valid in the interval $\phi \in[-\pi, \pi)$, in which the mean and the standard deviation have problems to correctly characterize interferometric phase statistics due to its circular nature [13].

Based on (2), Lee et al. [9] proved that the interferometric phase noise in the real domain can be characterized by an additive noise model

$$
\phi_{z}=\phi_{x}+v
$$

where $\phi_{z}$ is the measured phase; $\phi_{x}$ is the original phase without noise; and $v$ represents a zero-mean noise depending on $n$ and $|\rho|$. The original phase $\phi_{x}$ and the noise $v$ are assumed to be independent from each other. To reduce the interferometric phase noise $v$ in the real domain presents the problem of the phase jumps, i.e., points where the interferometric phase goes from $-\pi$ to $\pi$. From a statistical point of view, it is necessary to eliminate these phase jumps, through an unwrapping process, in order to correctly calculate the statistical parameters. In terms of noise reduction, as phase jumps represent high-frequency information, it is necessary to avoid its filtering in order to correctly unwrap the interferometric phase. To avoid these problems, the interferometric phase noise can be reduced in the complex domain, where the phase jumps are not present.

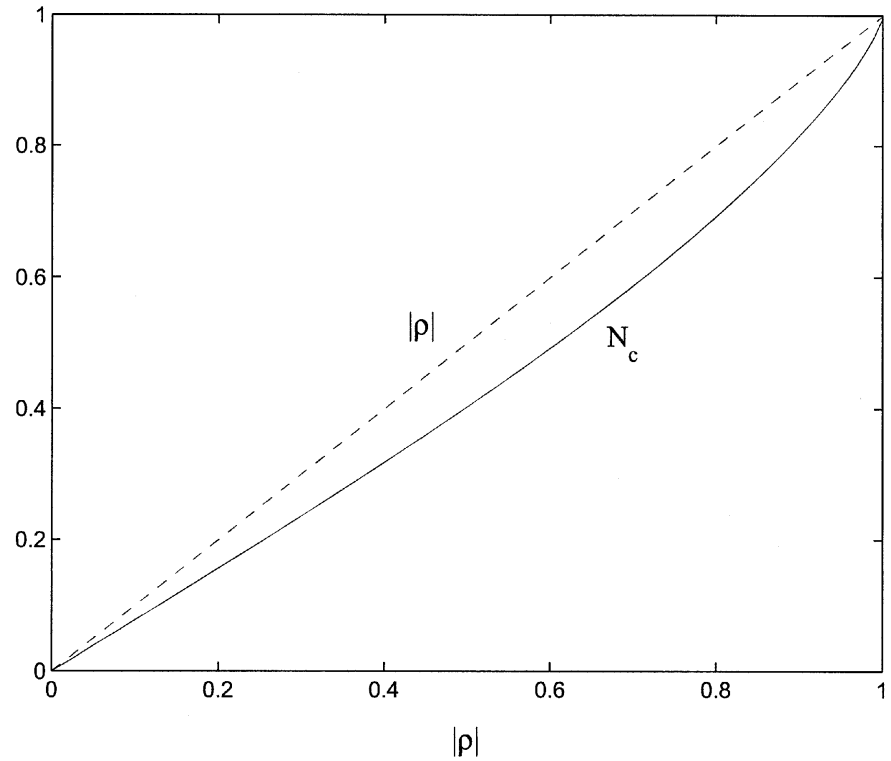

Fig. 1. Comparison between $N_{c}$ and $|\rho|$.

\section{A. Complex Interferometric Phase Noise Model}

In the complex domain, the interferometric phase is encoded as a point in the unitary circle

$$
e^{j \phi_{z}}=\cos \left(\phi_{z}\right)+j \sin \left(\phi_{z}\right) .
$$

In order to derive a noise model for (4), the real part is analyzed. For the imaginary part, only the final expression is given, as it can be derived in the same way. Based on (3), $\cos \left(\phi_{z}\right)$ can be written as

$$
\cos \left(\phi_{z}\right)=\cos \left(\phi_{x}+v\right)=\cos \left(\phi_{x}\right) \cos (v)-\sin \left(\phi_{x}\right) \sin (v)
$$

where the original signal $\phi_{x}$ has been separated from the noise contribution $v$. Alternative expressions for $\cos (v)$ and $\sin (v)$ can be obtained. Substituting $v_{1}=\cos (v)$ and $v_{2}=\sin (v)$ and making use of (2), the corresponding pdfs for $n=1$ (i.e., one-look), can be obtained in (6) and (7) (shown at the bottom of the page).

The mean values of $v_{1}$ and $v_{2}$, represented by $N_{c}$ and $N_{s}$ respectively, are given by

$$
\begin{aligned}
& N_{c}=\frac{\pi}{4}|\rho| F\left(\frac{1}{2}, \frac{1}{2} ; 2 ;|\rho|^{2}\right) \\
& N_{s}=0 .
\end{aligned}
$$

Fig. 1 shows the curve of $N_{c}$, where it can be observed that $N_{c}$ is monotone increasing with $|\rho|$. This property will allow to use

$$
\begin{aligned}
p_{v_{1}}\left(v_{1}\right) & =\frac{1}{\pi \sqrt{1-v_{1}^{2}}} \frac{\left(1-|\rho|^{2}\right)\left[\left(1-|\rho|^{2} v_{1}^{2}\right)^{1 / 2}+|\rho| v_{1}\left(\pi-\arccos \left(|\rho| v_{1}\right)\right)\right]}{\left(1-|\rho|^{2} v_{1}^{2}\right)^{3 / 2}}-1 \leq v_{1} \leq 1 \\
p_{v_{2}}\left(v_{2}\right) & =\frac{1}{\pi \sqrt{1-v_{2}^{2}}} \frac{\left(1-|\rho|^{2}\right)\left[\left(1-|\rho|^{2}\left(1-v_{2}^{2}\right)\right)^{1 / 2}+|\rho| \sqrt{1-v_{2}^{2}}\left(\frac{\pi}{2}-\arccos \left(|\rho| \sqrt{1-v_{2}^{2}}\right)\right)\right]}{\left(1-|\rho|^{2}\left(1-v_{2}^{2}\right)\right)^{3 / 2}} \\
-1 & \leq v_{2} \leq 1 .
\end{aligned}
$$


$N_{c}$ as a new interferometric phase quality parameter, instead of the coherence $|\rho|$. The variances of $v_{1}$ and $v_{2}$ are calculated numerically. Fig. 2 shows the variances of $v_{1}$ and $v_{2}$, referred as $\sigma_{v_{1}}^{2}$ and $\sigma_{v_{2}}^{2}$, respectively, as a function of the coherence. The noise contributions $v_{1}$ and $v_{2}$ can be represented by the addition of their means plus zero-mean random variables

$$
\begin{aligned}
& v_{1}=\cos (v)=N_{c}+v_{1}^{\prime} \\
& v_{2}=\sin (v)=N_{s}+v_{2}^{\prime} .
\end{aligned}
$$

Then, (5) can be rewritten as

$$
\cos \left(\phi_{z}\right)=N_{c} \cos \left(\phi_{x}\right)+v_{1}^{\prime} \cos \left(\phi_{x}\right)-v_{2}^{\prime} \sin \left(\phi_{x}\right)
$$

that can be expressed as

$$
\cos \left(\phi_{z}\right)=N_{c} \cos \left(\phi_{x}\right)+v_{c}
$$

where $v_{c}=v_{1}^{\prime} \cos \left(\phi_{x}\right)-v_{2}^{\prime} \sin \left(\phi_{x}\right)$. Using the same procedure, the expression for the imaginary part is obtained as

$$
\sin \left(\phi_{z}\right)=N_{c} \sin \left(\phi_{x}\right)+v_{s}
$$

where $v_{s}=v_{1}^{\prime} \sin \left(\phi_{x}\right)+v_{2}^{\prime} \cos \left(\phi_{x}\right)$. The first terms of (13) and (14) contain the original signal $\phi_{x}$ multiplied by the factor $N_{c}$. The second terms, $v_{c}$ and $v_{s}$, as it shall be demonstrated, can be considered as zero-mean additive noise processes. For the real part, (13), the variance of $v_{c}$ has the expression

$$
\begin{aligned}
\sigma_{v_{c}}^{2} & =E\left\{\left(v_{1}^{\prime} \cos \left(\phi_{x}\right)-v_{2}^{\prime} \sin \left(\phi_{x}\right)\right)^{2}\right\} \\
& =\sigma_{v_{1}}^{2} E\left\{\cos ^{2}\left(\phi_{x}\right)\right\}+\sigma_{v_{2}}^{2} E\left\{\sin ^{2}\left(\phi_{x}\right)\right\}
\end{aligned}
$$

where $E\left\{v_{1}^{\prime} v_{2}^{\prime}\right\}=0$. If the interferometric phase signal $\phi_{x}$ is considered as a random phase, then (15) leads to

$$
\sigma_{v_{c}}^{2}=\frac{1}{2}\left(\sigma_{v_{1}}^{2}+\sigma_{v_{2}}^{2}\right) .
$$

On the contrary, for a constant phase $\phi_{x},(16)$ does not hold. In this case, as $\sigma_{v_{c}}^{2}$ and $\sigma_{v_{s}}^{2}$ have similar values, (16) is taken as an approximation to the actual value. Fig. 2 shows the effect of this simplification. Using the same procedure, one can prove that $\sigma_{v_{s}}^{2}$ has the same expression as (16) (i.e., $\sigma_{v_{c}}^{2}=\sigma_{v_{s}}^{2}$ ). Therefore, $v_{c}$ and $v_{s}$ can be defined as statistically equal noise terms, and also independent from the original signal $\phi_{x}$. In this sense, (13) and (14) define a model for the interferometric phase noise in the complex domain. Accordingly, noise has a multiplicative behavior given by $N_{c}$ as well as an additive one given by the noise terms $v_{c}$ and $v_{s} . N_{c}$ and the noises $v_{c}$ and $v_{s}$ are not independent, as they depend on $|\rho|$. With an increasing of $|\rho|, N_{c}$ increases, and $\sigma_{v_{c}}^{2}$ and $\sigma_{v_{s}}^{2}$ decrease.

To prove the validity of the model, mean-standard deviation scatter plots have been calculated using simulated as well as real interferometric phases. As at this point, spatial resolution is not considered, the original signal $\cos \left(\phi_{x}\right)$ is calculated applying a multilook filter with a $7 \times 7$ pixel window [10]. The mean and the standard deviation of $\cos \left(\phi_{z}\right)$ are calculated using 7 $\times 7$ pixel nonoverlapped windows. First, a simulated interferometric phase ramp of $1024 \times 1024$ pixels, with wide fringes in order to avoid problems calculating the statistics, has been employed. Fig. 3(a) shows the mean-standard deviation scatter plot for the real part of the simulated complex phase with a co-

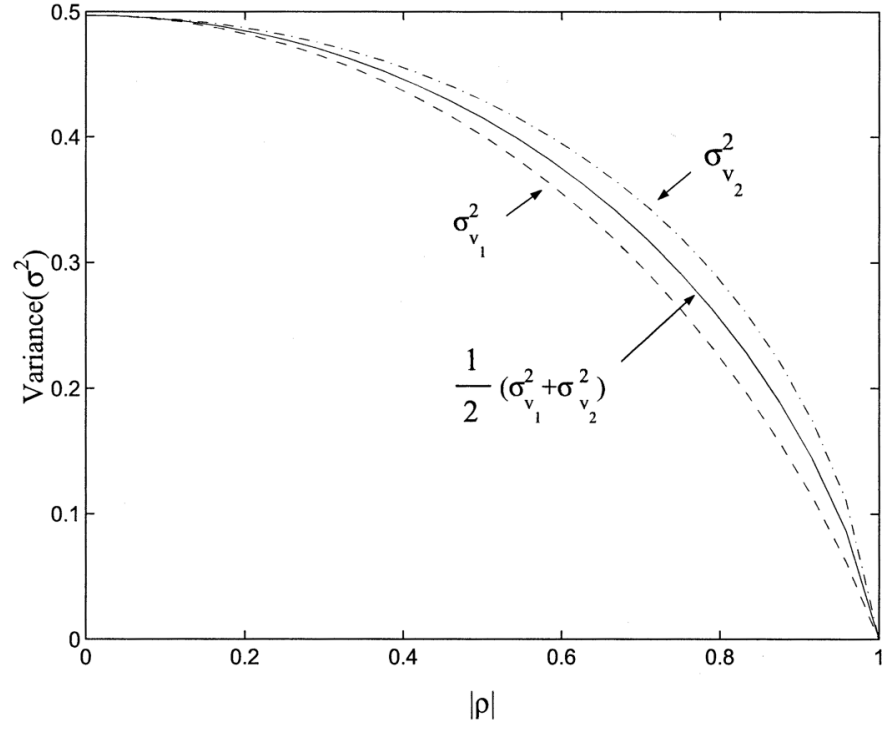

Fig. 2. Complex interferometric phase noise variances.

herence of 0.7. The dashed lines represent the theoretical relation between mean and standard deviation of $\cos \left(\phi_{z}\right)$, for those cases where $\cos \left(\phi_{x}\right)$ is equal to $0, \pm 0.5, \pm \sqrt{3} / 2$, and \pm 1 . The plots show that the value $\sigma_{v_{c}}$ is almost independent of the original signal value $\cos \left(\phi_{x}\right)$, as stated in (16). The curvature at the extremes is due to the dependence between $N_{c}$ and $\sigma_{v_{c}}$, as both of them depend on $|\rho|$. Fig. 3(b) presents the mean-standard deviation scatter plot for a simulated constant phase of value $\phi_{x}=\pi / 4 \mathrm{rad}$, for coherence values between 0.5 and 1 . The white dashed line represents the theoretical mean-standard deviation relation of $\cos \left(\phi_{z}\right)$. In this case, the simulated data fit completely the theoretical curve. Fig. 3(c) represents a $1024 \times$ 1024 pixel image segment of an X-band interferometric phase of Mount Etna, Sicily, with $3 \times 3-\mathrm{m}$ spatial resolution, acquired by the E-SAR system of the German DLR. Fig. 3(d) shows that the real statistics follow the theoretical relation between the mean and the standard deviation of $\cos \left(\phi_{z}\right)$. Again, for clarity reasons, the theoretical relation between the mean and the standard deviation of $\cos \left(\phi_{z}\right)$ is plotted with dashed lines for the cases where $\cos \left(\phi_{x}\right)$ is equal to $0.5,0.9$, and 1 . Although statistics for the imaginary part are not presented, they also present the same agreement with the theoretical model.

\section{B. Complex Wavelet Interferometric Phase Noise Model}

The interferometric phase noise model in the complex domain, introduced in Section II-A, will now be addressed in the wavelet domain, as within this domain an efficient use of it can be performed. The continuous wavelet transform of a function $f(t) \in L^{2}(\mathbf{R})$ is defined as the integral on $L^{2}(\mathbf{R})$ [14]

$$
\mathrm{WT} f(u, s)=\int_{-\infty}^{\infty} f(t) \psi_{u, s}^{*}(t) d t
$$

where the functions $\psi_{u, s}(t) \in L^{2}(\mathbf{R})$ are defined as

$$
\psi_{u, s}(t)=\frac{1}{\sqrt{s}} \psi\left(\frac{t-u}{s}\right)
$$

with $s, u \in \mathbf{R}$ and $s \neq 0$. The function $\psi$ is called the mother wavelet, whereas the family $\psi_{u, s}(t)$ are called wavelets. The 


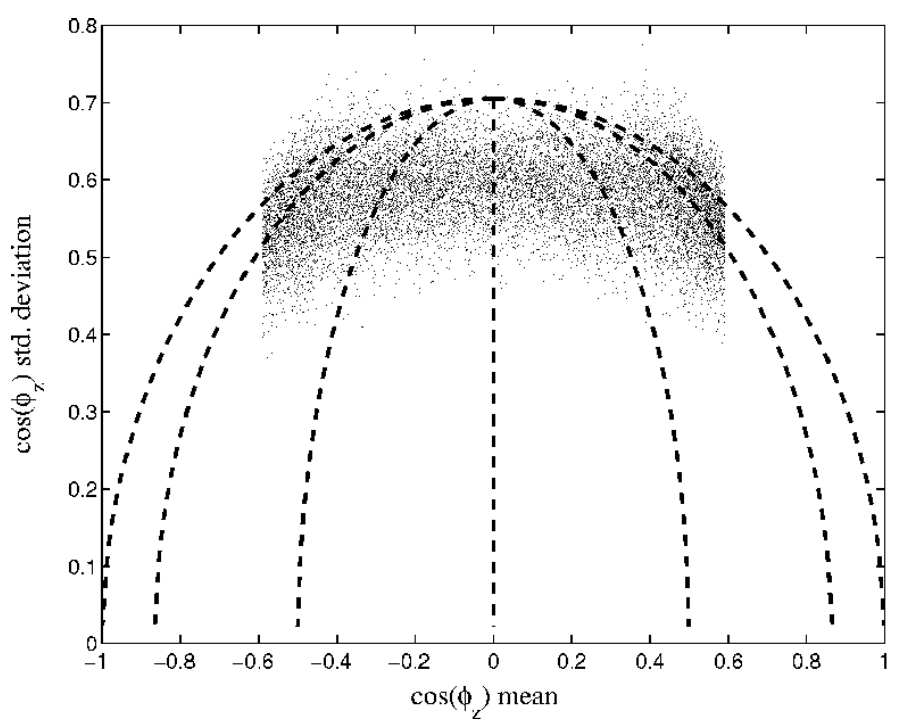

(a)

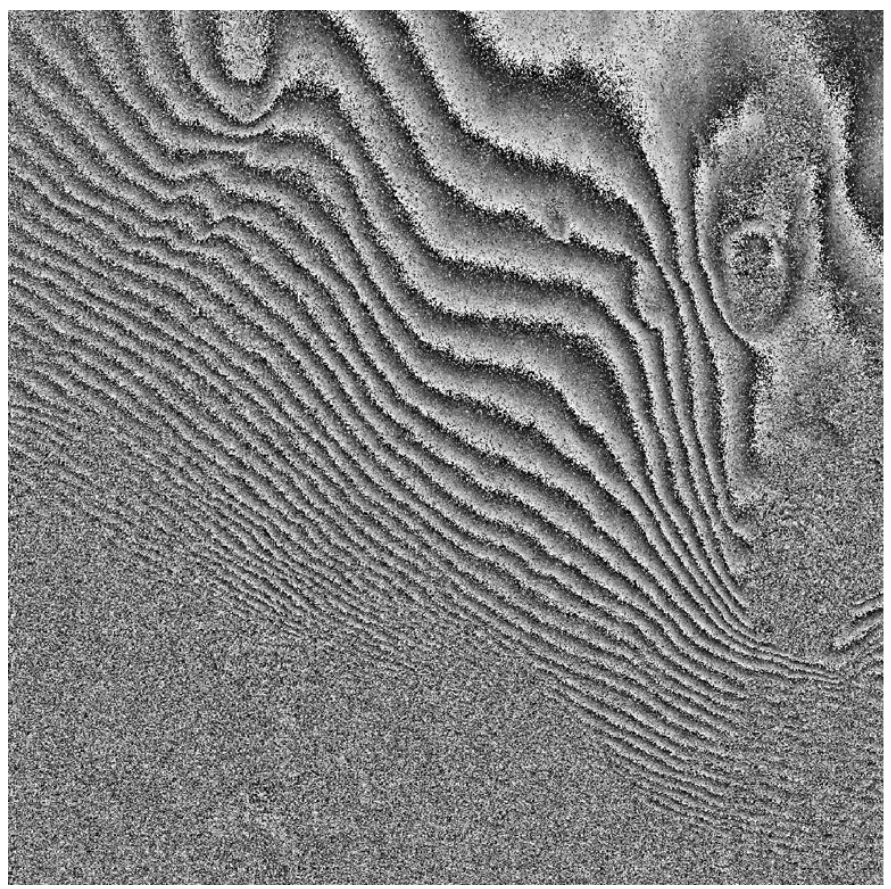

(c)

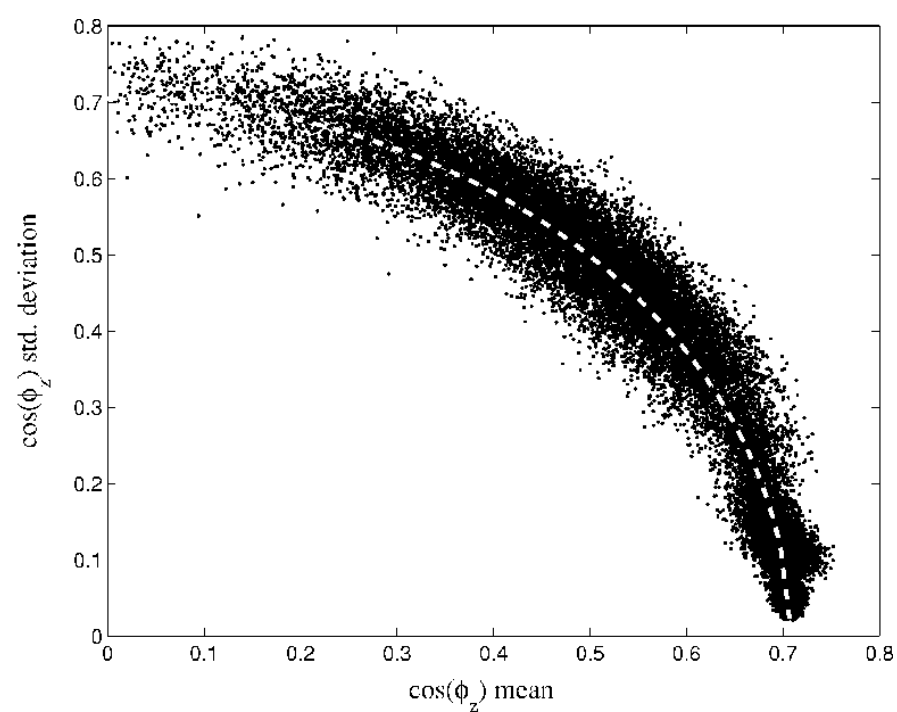

(b)

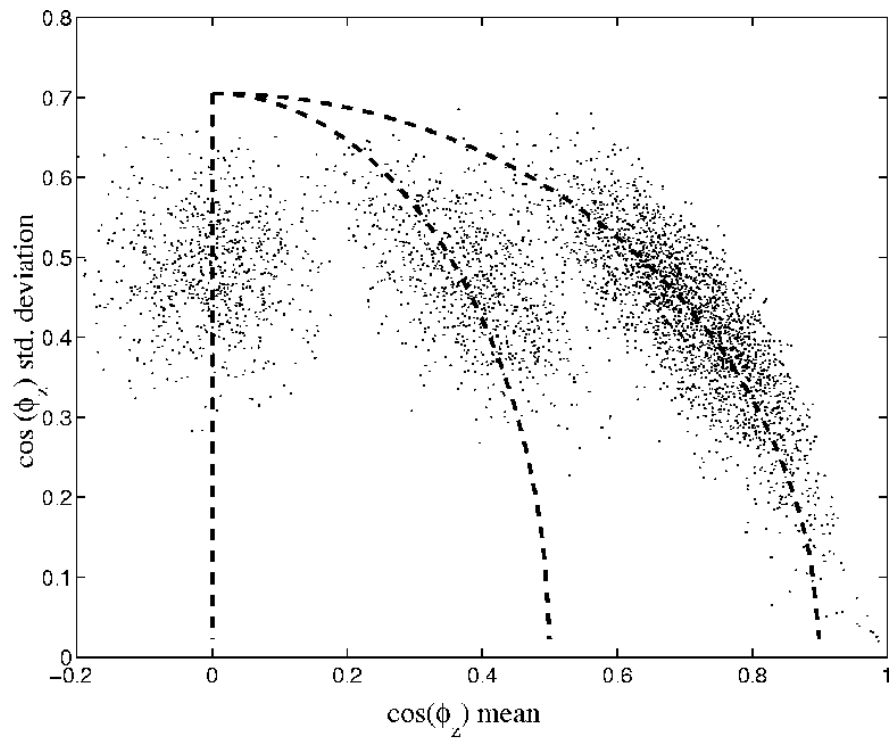

(d)

Fig. 3. Mean-standard deviation scatter plots for interferometric phase noise. (a) Scatter plot of $\cos \left(\phi_{z}\right)$ for a simulated phase ramp $|\rho|=0.7$. (b) Scatter plot of $\cos \left(\phi_{z}\right)$ for a constant phase $\phi_{x}=\pi / 4$, with coherences from 0.5 to 1. (c) Interferometric phase of Mount Etna. (d) Scatter plots of Mount Etna phase for some values of $\cos \left(\phi_{x}\right)$.

wavelet transform (WT) defines a two-dimensional (2-D) transformed domain for one-dimensional (1-D) signals. The parameter $s$ (scale), controlling dilatations of $\psi$, is related to frequency information [15]. The parameter $u$ (time), controlling shifts of $\psi$, gives information about time. The Mallat algorithm [14] proves that a fast discrete wavelet transform (DWT) can be calculated by using a filter bank based on the iteration of a cell of filters: the pair $\bar{h}, \bar{g}$ for the DWT process, and the pair $h, g$ for the inverse DWT, where $\bar{h}[n]=h[-n]$. Each time the cell of filters is iterated, a new wavelet scale is obtained. Each of the discrete wavelet coefficients contains, then, information about a particular space-frequency area of the original signal [16]. Fig. 4 presents a scheme of the process to calculate the DWT and the inverse DWT for 1-D signals. The bands $a_{i}$, also called residue bands, contain low-frequency information, as their frequency support is $\omega \in\left[0,2^{-i} \pi\right)$ for the scale $2^{i}$. On the contrary, the bands $d_{i}$, or wavelet bands, contain high-frequency information, as their frequency support is $\omega \in\left[2^{-i} \pi, 2^{1-i} \pi\right)$ for the scale $2^{i}$. The 2-D DWT can be calculated in a separable way applying the scheme given by Fig. 4 to each of the spatial dimensions [14]. In this case, the DWT is composed in each scale $2^{i}$ by a residue band $a_{i}^{\mathrm{LL}}$ and by three wavelet bands $d_{i}^{\mathrm{HL}}, d_{i}^{\mathrm{LH}}$, and $d_{i}^{\mathrm{HH}}$.

As the WT is a linear operation, the noise model defined by (13) and (14) is maintained within the wavelet domain. In this domain, the noise model expressions can be derived assuming 


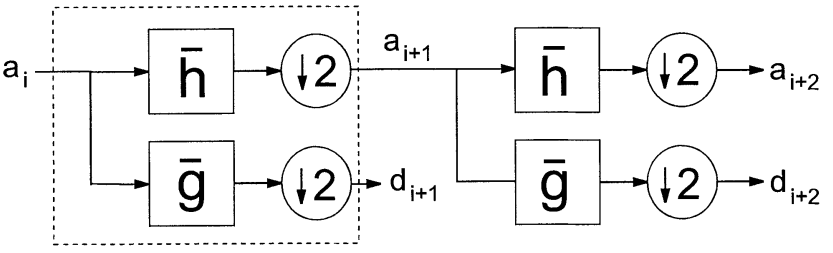

(a)

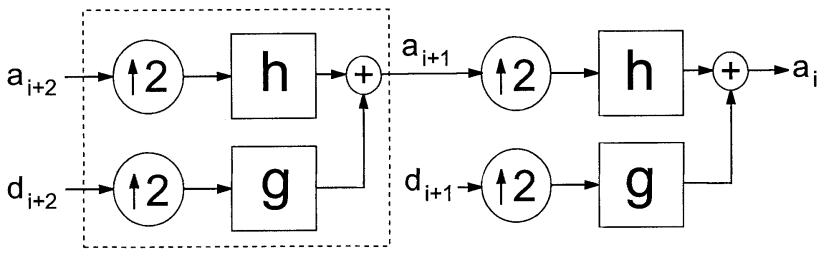

(b)

Fig. 4. Scheme for fast DWT. (a) DWT. (b) Inverse DWT.

ideal filters for the DWT. This assumption holds if the DWT is calculated using the Shannon wavelet [17], whose filters response are given by

$$
\begin{aligned}
& H(\omega)= \begin{cases}\alpha, & |\omega| \in\left[0,2^{-1} \pi\right) \\
0, & \text { otherwise }\end{cases} \\
& G(\omega)= \begin{cases}\alpha, & |\omega| \in\left[2^{-1} \pi, \pi\right) \\
0, & \text { otherwise }\end{cases}
\end{aligned}
$$

where $H(\omega)$ and $G(\omega)$ are the frequency responses of the filters $h$ and $g$, respectively, and $\alpha$ represents the filter amplitude. The necessary and sufficient conditions required for any filter to synthesize orthogonal wavelets are [14]

$$
\forall \omega \in \mathbf{R}|H(\omega)|^{2}+|H(\omega+\pi)|^{2}=2
$$

with

$$
H(0)=\sqrt{2} .
$$

Using (20) and (21), $\alpha=\sqrt{2}$ is obtained. For the 1-D DWT, the total response at the residue bands $a_{i}$ can be viewed as a cascade of a number $i$ of filters with response $\bar{h}$, each one followed by subsampling by two. This structure is equivalent to a filter with an amplitude equal to $\alpha^{i}=2^{i / 2}$ and a frequency support $\omega \in\left[0,2^{-i} \pi\right)$, followed by a subsampling by $2^{i}$. For the sake of simplicity, the terrain will be considered as a constant slope. In this case, therefore, the original signal $\phi_{x}$ can be assumed to be a linear ramp (i.e., $\cos \left(\phi_{x}\right)=\cos \left(\omega_{x} n\right)$, where $\omega_{x}$ is the original signal, and $n$ is the spatial coordinate) [18], and (13) can be expressed as

$$
\cos \left(\phi_{z}\right)=N_{c} \cos \left(\omega_{x} n\right)+v_{c} .
$$

Applying the DWT, calculated with the Shannon wavelet, the wavelet transform of (22) into the residue bands $a_{i}$, assuming $\omega_{x} \in\left[0,2^{-i} \pi\right)$, has the expression

$$
\operatorname{DWT}_{1 D}\left\{\cos \left(\phi_{z}\right)\right\}=2^{i / 2} N_{c} \cos \left(2^{i} \omega_{x} n\right)+v_{c}^{w}
$$

where $v_{c}^{w}$ represents the DWT of the noise term $v_{c}$ at the scale $2^{i}$. As the noise $v_{c}$ has zero mean, $v_{c}^{w}$ is also a zero-mean noise. Assuming the local power spectra of $v_{c}$ to be equal to

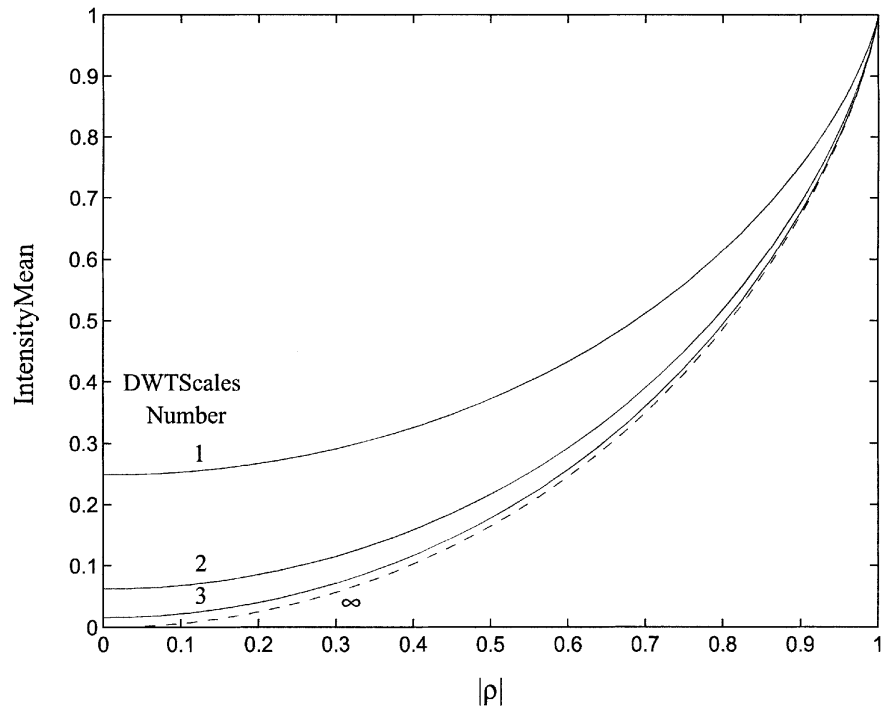

Fig. 5. Effect of the number of wavelet scales in the wavelet coefficients intensity mean. Intensities are normalized by $2^{2 i}$.

$S_{v_{c} v_{c}}(\omega)=\sigma_{v_{c}}^{2}$, the variance of $v_{c}^{w}$ (assuming again an ideal DWT) at any wavelet scale $2^{i}$ has the expression

$$
\begin{aligned}
\sigma_{v_{c}^{w}}^{2} & =\frac{1}{2 \pi} \int_{-\pi}^{\pi} S_{v_{c} v_{c}}(\omega)\left|H_{i}(\omega)\right|^{2} d \omega \\
& =\frac{1}{2 \pi} \int_{-\pi}^{\pi} \sigma_{v_{c}}^{2} 2^{i} 1_{\left[-2^{-i} \pi, 2^{-i} \pi\right]}(\omega) d \omega=\sigma_{v_{c}}^{2} .
\end{aligned}
$$

Equation (23) states that for each new wavelet scale $2^{i}$, the DWT increases the original signal component by a factor $2^{1 / 2}$, without varying the power's noise, as the variance of $v_{c}^{w}$ has the same value as the variance of $v_{c}$. The noise model expression of the imaginary part, within the bands $a_{i}$, assuming $\omega_{x} \in\left[0,2^{-i} \pi\right)$, is

$$
\operatorname{DWT}_{1 D}\left\{\sin \left(\phi_{z}\right)\right\}=2^{i / 2} N_{c} \sin \left(2^{i} \omega_{x} n\right)+v_{s}^{w} .
$$

As within the original domain, the noise terms $v_{c}^{w}$ and $v_{s}^{w}$ are statistically equal, but also independent from the wavelet scale $2^{i}$.

Using a similar procedure, it can be shown that if the original signal $\omega_{x}$ is located in a frequency band $d_{i}$, i.e., $\omega_{x} \in$ $\left[2^{-i} \pi, 2^{1-i} \pi\right)$, the noise model becomes slightly different. In this case, the expressions for the transformed real and imaginary parts of the noise model are

$$
\begin{aligned}
& \operatorname{DWT}_{1 D}\left\{\cos \left(\phi_{z}\right)\right\}=2^{i / 2} N_{c} \cos \left(\left(2 \pi-2^{i} \omega_{x}\right) n\right)+v_{c}^{w} \\
& \operatorname{DWT}_{1 D}\left\{\sin \left(\phi_{z}\right)\right\}=2^{i / 2} N_{c} \sin \left(\left(2 \pi-2^{i} \omega_{x}\right) n\right)+v_{s}^{w} .
\end{aligned}
$$

First addend of (23), (25)-(27) contains information about the original signal $\omega_{x}$. When the signal $\omega_{x}$ is contained in a frequency range of a band $d_{j}$, different from $a_{i}$ or $d_{i}$, or there is no signal in any band (i.e., $|\rho|=0$ ), the noise model for the real and imaginary parts in the band $a_{i}$ or $d_{i}$ has the expressions

$$
\begin{aligned}
& \operatorname{DWT}_{1 D}\left\{\cos \left(\phi_{z}\right)\right\}=v_{c}^{w} \\
& \operatorname{DWT}_{1 D}\left\{\sin \left(\phi_{z}\right)\right\}=v_{s}^{w} .
\end{aligned}
$$

In this case, the noise terms $v_{c}^{w}$ and $v_{s}^{w}$ have a variance equal to 0.5 (see Fig. 2). 


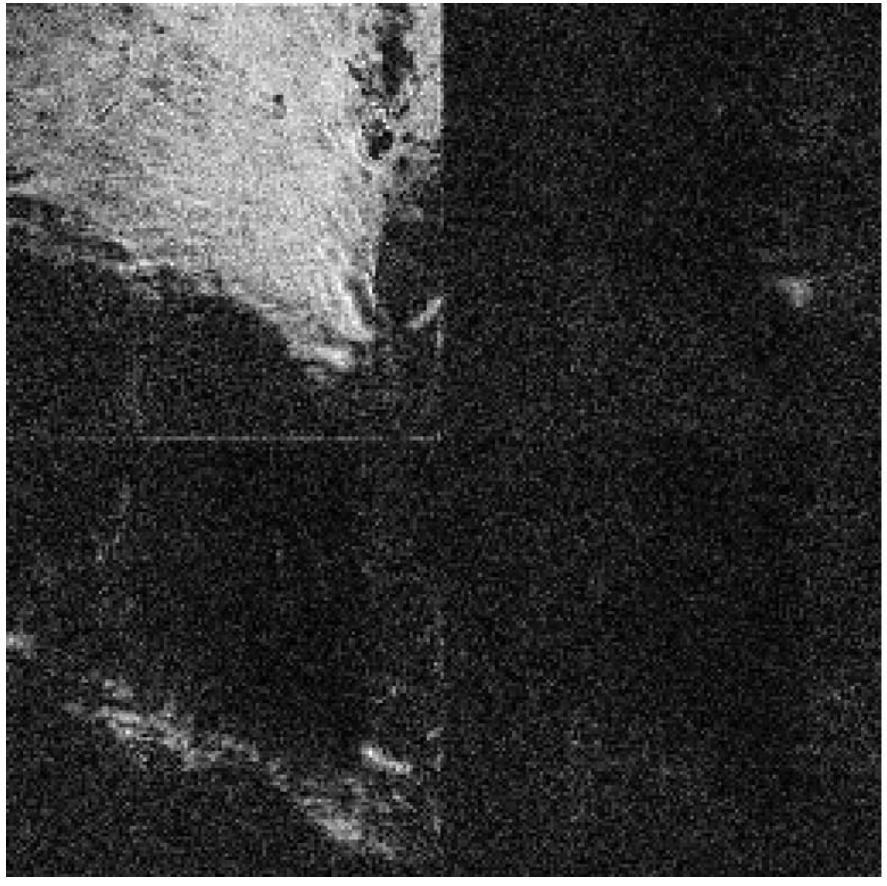

(a)

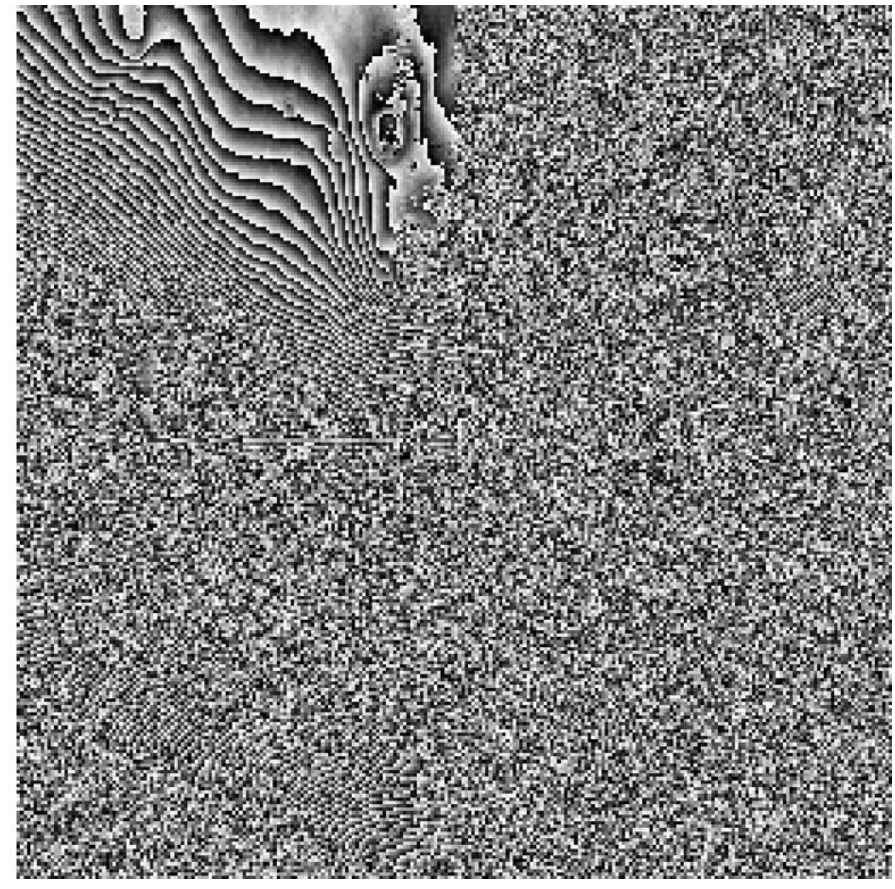

(b)

Fig. 6. DWT of the interferometric phase image Fig. 3(c). From left to right and from top to bottom $a_{3}^{\mathrm{LL}}, d_{3}^{\mathrm{HL}}, d_{3}^{\mathrm{LH}}$, and $d_{3}^{\mathrm{HH}}$ wavelet bands are shown. (a) Amplitude. (b) Phase. Amplitude is displayed for better contrast.

The DWT performs a frequency division, locating the signal $\omega_{x}$ in the different frequency bands, $a_{i}$ or $d_{i}$. As there exist spatial resolution inside the bands $a_{i}$ or $d_{i}$, spatial location information of the signal $\omega_{x}$, in a particular wavelet scale $2^{i}$, can be obtained also. In this noise model, $N_{c}$ plays an important role. For $N_{c}$ equal to zero, it means that there is only noise in a particular space-frequency area defined by the corresponding wavelet coefficient [(28) and (29)]. For values greater than zero, $N_{c}$ indicates that there is useful signal content in a particular position in the wavelet domain, but also gives information about the signal quality in that area. Therefore, $N_{c}$ allows to locate wavelet coefficients containing information about the signal $\omega_{x}$.

SAR interferometric phases are 2-D images. In this case, the noise model is slightly different. It can be proved that the only change is that the first addend of the noise model equations are multiplied by a factor of two instead of $2^{1 / 2}$ each time a wavelet scale is calculated, thus having a larger signal improvement. The noise model equations, for a complex interferometric phase image in the wavelet domain, have the expressions

$$
\begin{aligned}
& \operatorname{DWT}_{2 D}\left\{\cos \left(\phi_{z}\right)\right\}=2^{i} N_{c} \cos \left(\phi_{x}^{w}\right)+v_{c}^{w} \\
& \mathrm{DWT}_{2 D}\left\{\sin \left(\phi_{z}\right)\right\}=2^{i} N_{c} \sin \left(\phi_{x}^{w}\right)+v_{s}^{w}
\end{aligned}
$$

where $\phi_{x}^{w}$ represents the phase information within the complex wavelet domain. For low-frequency bands (i.e., $a_{i}^{\mathrm{LL}}$ ), this phase is a filtered and downsampled version of the original interferometric phase $\phi_{x}$. For high-frequency bands (i.e., $d_{i}^{\mathrm{HL}}, d_{i}^{\mathrm{LH}}$, and $\left.d_{i}^{\mathrm{HH}}\right) \phi_{x}^{w}$ is, on the contrary, a filtered, downsampled, and also frequency-inverted version of the original interferometric phase $\phi_{x}$.
Equations (30) and (31) define a complex wavelet domain. Inside this domain, the intensity and the phase information of a wavelet coefficient have a more important role than the real and imaginary parts. The wavelet coefficient intensity is the most important parameter, as it gives information about noise content and since it can be directly related with the parameter $N_{c}$. The mean of the intensity for a wavelet coefficient has the expression

$$
E\left\{\left|\mathrm{DWT}_{2 D}\left(e^{j \phi_{z}}\right)\right|^{2}\right\}=2^{2 i} N_{c}^{2}+\sigma_{v_{c}^{w}}^{2}+\sigma_{v_{s}^{w}}^{2} .
$$

When the wavelet coefficient contains only noise (i.e., $N_{c}=0$ ), its value is reduced to

$$
E\left\{\left|\operatorname{DWT}_{2 D}\left(e^{j \phi_{z}}\right)\right|^{2}\right\}=\sigma_{v_{c}^{w}}^{2}+\sigma_{v_{s}^{w}}^{2} .
$$

As $0 \leq N_{c} \leq 1$ (Fig. 1) and $\left(\sigma_{v_{c}^{w}}^{2}+\sigma_{v_{s}^{w}}^{2}\right) \leq 1$ (Fig. 2), for $2^{2 i}$ large enough, $\sigma_{v_{c}^{w}}^{2}+\sigma_{v_{s}^{w}}^{2}$ is negligible compared with $2^{2 i} N_{c}^{2}$. Hence, (32) can be written as $2^{2 i} N_{c}^{2}$, which is monotone increasing with the coherence $|\rho|$ (Fig. 1). Fig. 5 shows (32), normalized by a factor $2^{2 i}$ in order to compare the effect of several wavelet scales. For three wavelet scales, the variances of the noise terms $v_{c}^{w}$ and $v_{s}^{w}$ are negligible compared with the factor $2^{2 i} N_{c}^{2}$.

The phase of the wavelet coefficients $\phi_{x}^{w}$ is defined as the wavelet interferometric phase. Using (30) and (31), the interferometric wavelet phase can be obtained, for $2^{i} N_{c}$ large enough, as

$\arg \left[\operatorname{DWT}_{2 D}\left(e^{j \phi_{z}}\right)\right]=\arctan \left(\frac{2^{i} N_{c} \sin \left(\phi_{x}^{w}\right)+v_{s}^{w}}{2^{i} N_{c} \cos \left(\phi_{x}^{w}\right)+v_{c}^{w}}\right) \approx \phi_{x}^{w}$ 


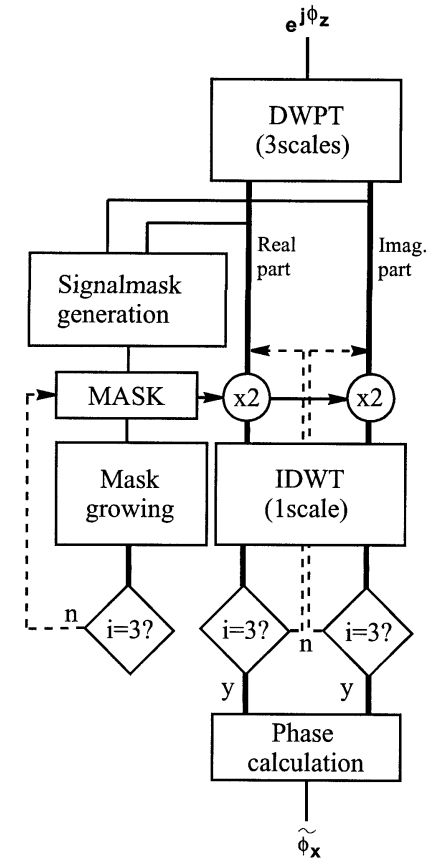

Fig. 7. Noise reduction algorithm flow diagram. Dashed lines represent iteration.

where $\arg \rrbracket$ represents the argument of a complex quantity. The resulting phase of the wavelet coefficient $\phi_{x}^{w}$ contains information about the original interferometric phase. For those wavelet coefficients having $N_{c}$ equal to zero, its phase contains only noise

$$
\arg \left[\operatorname{DWT}_{2 D}\left(e^{j \phi_{z}}\right)\right]=\arctan \left(\frac{v_{s}^{w}}{v_{c}^{w}}\right) .
$$

Fig. 6 presents the amplitude and the phase of the DWT of the interferometric phase of Mount Etna already shown in Fig. 3(c). It can be noticed that those wavelet coefficients having high amplitude contain information about the original interferometric phase, whereas those wavelet coefficients with low amplitude only contain noise in the phase.

The DWT is able to locate the interferometric phase signal in a space-frequency domain. The noise model developed in this section [(30) and (31)] shows the intrascale model of wavelet coefficients for the real and imaginary parts, i.e., the sort of information that each wavelet coefficient gives. In [19], it was shown that the wavelet coefficients in different scales can be related hierarchically, as they are a function of the same spatial area of the original image. The topographic signal can be assumed locally as a narrow-frequency band signal [20] (i.e., linear phase); then, for all the wavelet coefficients describing the same area, only those in the frequency scale containing the original signal will have large intensity values, whereas the coefficients in the rest of scales, referring to the same area, will contain only noise. For topographic information, the useful signal is mainly concentrated in the frequency range $\omega \in\left[0,2^{-1} \pi\right)$, whereas the range $\omega \in\left[2^{-1} \pi, \pi\right]$ contains only noise; then, the DWT concentrates the useful signal in the lower frequency part of the spectra. In Fig. 6, this effect can be seen; high-frequency fringes are also concentrated in low-frequency bands. Consequently, the overall
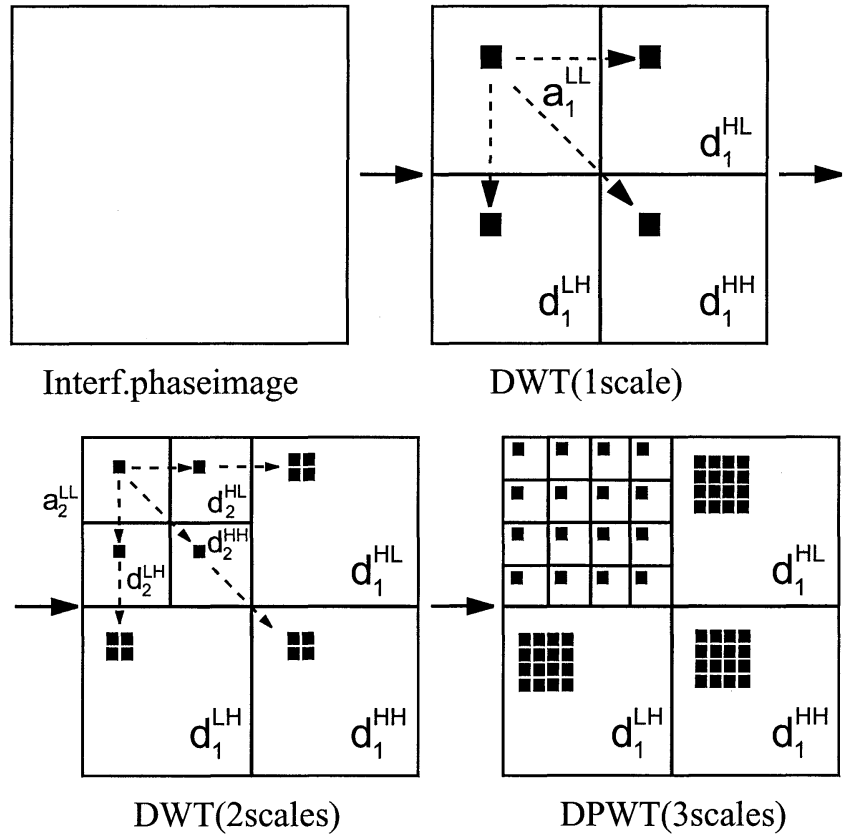

Fig. 8. Wavelet transformation process employed to transform interferometric complex phase images. Relations between pixels (black boxes) in different scales are shown.

behavior of the wavelet coefficients can be summarized as follows.

- The position in the scale dimension gives frequency information about the interferometric phase signal.

- The position in the space dimension gives spatial location about the frequency content.

- The coefficient value gives information in a local spacefrequency area. If the intensity has a high value, i.e., a high value of $N_{c}$, then the phase contains information about topography. These coefficients are defined as signal coefficients. On the contrary, if the intensity is low, i.e., $N_{c}$ is equal to zero, then the phase contains only noise. These coefficients are defined as noise coefficients.

The interferometric phase noise model in the wavelet domain [(30) and (31)] have been derived assuming an idealization of the DWT, employing a Shannon wavelet as a wavelet filter. The noise model is completely valid when other wavelet filters are used, due to the fact that all the filters have to fulfill the conditions given by (20) and (21). On the contrary, using the developed interferometric phase noise model equations, it can be demonstrated that complex wavelet filters cannot be employed, as these filters introduce an additional phase term depending both on the wavelet filter and on the signal $\phi_{x}$.

\section{NoIse Reduction ALGORITHM}

In this section, a new approach to remove interferometric phase noise in the wavelet complex domain, based on the noise model introduced in Section II, is presented. As stated, the DWT multiplies the original signal term by a factor of two, without altering the noise power, each time a wavelet scale is performed. This improvement is lost when the IDWT is applied. The task of the algorithm will be to maintain this improving factor by avoiding the loss in the inverse 
TABLE I

Interferometric Phase Filters Performance Comparison. "MSE r.p." Refers to the mse Performed in the Real Plane, Whereas "MSE c.p." Refers to THE mse CALCULATED IN THE COMPLEX Plane

\begin{tabular}{l|l|r|r|r|r}
\multicolumn{2}{c}{} & WInPF filter & Multilook fil. & FFT filter & Lee filter \\
\hline \multirow{5}{*}{$|\rho|=0.9$} & 0.788 & 1.461 & 1.125 & 0.832 \\
& MSE r.p. $\left(\mathrm{rad}^{2}\right)$ & 0.032 & 0.090 & 0.056 & 0.062 \\
& MSE c.p. $\left(\mathrm{rad}^{2}\right)$ & 0 & 2 & 16 & 107 \\
& Num. residues & 15.3 & 0.9 & 5.1 & 565.6 \\
\hline \multirow{5}{*}{$|\rho|=0.7$} & Exec. time (s) & 1.357 & 1.820 & 1.109 & 1.805 \\
& MSE r.p. $\left(\mathrm{rad}^{2}\right)$ & 0.162 & 0.298 & 0.314 \\
& MSE c.p. $\left(\mathrm{rad}^{2}\right)$ & 094 & 94 & 798 & 936 \\
& Num. residues & 105 & 0.4 & 5.5 & 593.4 \\
\hline \multirow{5}{*}{$|\rho|=0.5$} & Exec. time (s) & 15.2 & 2.608 & 3.785 & 3.145 \\
& MSE r.p. $\left(\mathrm{rad}^{2}\right)$ & 2.102 & 0.341 & 1.084 & 0.968 \\
& MSE c.p. $\left(\mathrm{rad}^{2}\right)$ & 0.230 & 1125 & 7200 & 2997 \\
& Num. residues & 694 & 0.4 & 4.5 & 574.9 \\
\hline
\end{tabular}

transformation process. As the intensity of the complex wavelet coefficients can be assumed monotone increasing with $|\rho|$, the algorithm detects the wavelet coefficients containing useful information and multiplies their real and imaginary parts by two before inversely transforming a wavelet scale. Therefore, the phase of the coefficients detected as signal coefficients will be enhanced in the inverse transformation process compared with the phase of the noise coefficients.

In a complex number, the phase information is contained in the relation between real and imaginary parts. Accordingly, when a complex signal is processed, phase preservation requires real and imaginary parts to be processed in the same way and by the same amount. As the phase noise is reduced in the complex plane, no phase unwrapping is required in the implementation of the algorithm.

The algorithm has been designed to maintain the spatial resolution without employing a windowing process, applying, on the contrary, the DWT to the whole image. In order to perform a local analysis of the original signal and the noise, the DWT frequency-space location property is employed [14]. As the algorithm is not based on square windows, there is no loss of resolution due to restrictions in the interval of processed frequencies. The main point in order to maintain the spatial resolution is the way in which the wavelet coefficients are processed. In an interferometric phase from medium to low coherence, it is difficult to determine if a phase detail is due to the original signal or comes from the noise. Then, instead of reducing, or even to eliminate noise wavelet coefficients, that can lead to a loss of resolution, the algorithm is based on increasing the importance of the wavelet signal coefficients. Acting in this way, we avoid losing possible information about details that may be included also within noise wavelet coefficients.

\section{A. Algorithm Implementation}

In the following, a step-by-step description of the algorithm used to remove interferometric phase noise in the complex plane, based on the WT, is provided, whereas a flow diagram of it is shown in Fig. 7.

Step 1 (DWT): The complex interferometric phase signal (4) is transformed to the wavelet domain. The wavelet transform is applied with three wavelet scales, as additive noise effects are then negligible in the wavelet coefficients intensity. After transforming the complex phase with a two-scale DWT, the signal is divided in the residue band $a_{2}^{\mathrm{LL}}$ and the wavelet bands $d_{2}^{\mathrm{HL}}, d_{2}^{\mathrm{LH}}$, $d_{2}^{\mathrm{HH}}, d_{1}^{\mathrm{HL}}, d_{1}^{\mathrm{LH}}$, and $d_{1}^{\mathrm{HH}}$. As said before, the bands $d_{1}^{\mathrm{HL}}, d_{1}^{\mathrm{LH}}$, and $d_{1}^{\mathrm{HH}}$ contain only noise, whereas the useful signal is concentrated in the rest of the bands, which will be called signal bands in the following. In order to obtain a constant amplification by a factor of $2^{2 i}$ (with $i=3$ ) for the intensity of those wavelet signal coefficients within the frequency range $\left[0,2^{-1} \pi\right)$, the discrete wavelet packet transform [21] is employed instead of the DWT. Therefore, to obtain the third wavelet scale, the wavelet filter cell is iterated, not only in the band $a_{2}^{\mathrm{LL}}$, as the DWT would do, but also in the bands $d_{2}^{\mathrm{HL}}, d_{2}^{\mathrm{LH}}$, and $d_{2}^{\mathrm{HH}}$. Fig. 8 shows a scheme about how the wavelet transform is applied for a given interferometric phase signal.

Step 2 (Signal Detection): To detect which wavelet coefficients in the frequency range $\left[0,2^{-1} \pi\right)$ contain useful information, the parameter $\Gamma_{\text {sig }}$, depending on the signal quality, is calculated

$$
\Gamma_{\text {sig }}=\frac{I_{w}-2^{2 i} \sigma_{w}^{2}}{I_{w}} .
$$

$I_{w}$ represents the intensity for those wavelet coefficients in the frequency range $\left[0,2^{-1} \pi\right)$, where the useful signal is concentrated. $\sigma_{w}^{2}$ is the noise variance in the same spatial area described by $I_{w}$. This noise variance can be also calculated from the intensity of wavelet coefficients, but in the frequency range $\left[2^{-1} \pi, \pi\right)$. In this case, the intensity mean, as only noise is present, has the value given by (33). The factor $2^{2 i}$ multiplying $\sigma_{w}^{2}$ compensates this value in $I_{w}$, according to (32). As coefficients in the bands $d_{1}^{\mathrm{HL}}, d_{1}^{\mathrm{LH}}$, and $d_{1}^{\mathrm{HH}}$ have a space relation of 16 to 1 with the wavelet coefficients in the rest of the bands, or signal bands, (see Fig. 8), $\sigma_{w}^{2}$ can be calculated as one half of the mean intensity of the 48 coefficients in the noise bands. Due to the orthogonality properties of the DWT, good estimation of noise variance is obtained. Now, a 1-to-1 space relation between $\sigma_{w}^{2}$ and the wavelet signal bands is obtained, making possible to calculate $\Gamma_{\text {sig }}$ for each of the 16 signal bands. If a wavelet coefficient is noise free, $\Gamma_{\text {sig }}$ equals one. As $\sigma_{w}^{2}$ increases, $I_{w}$ 


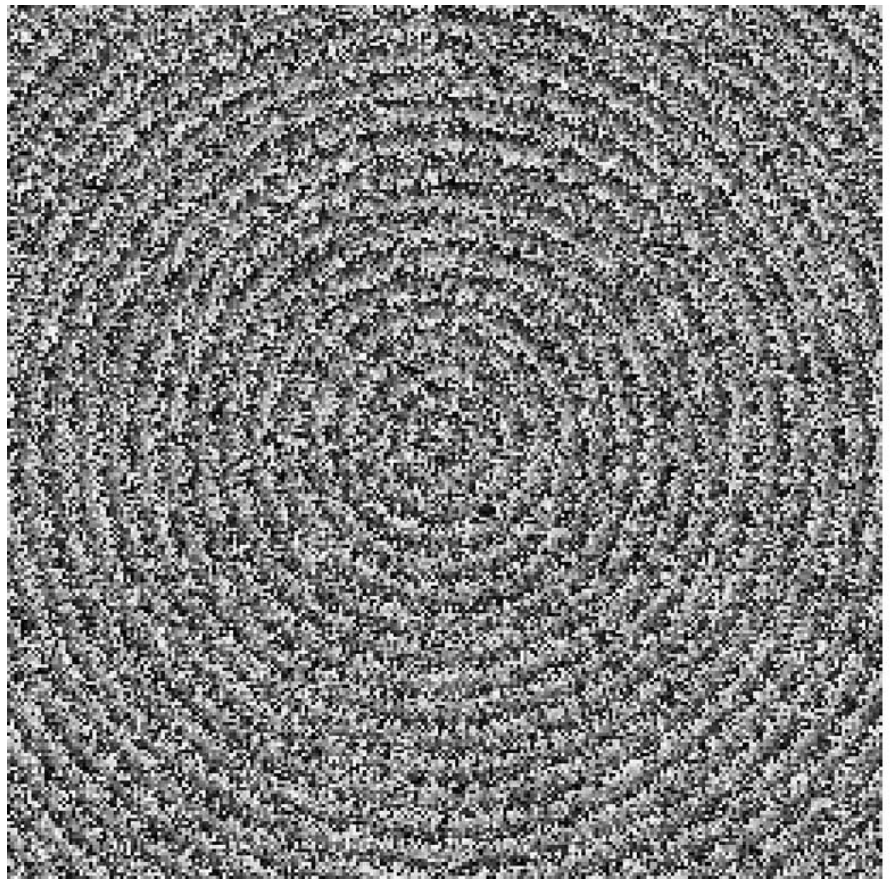

(a)

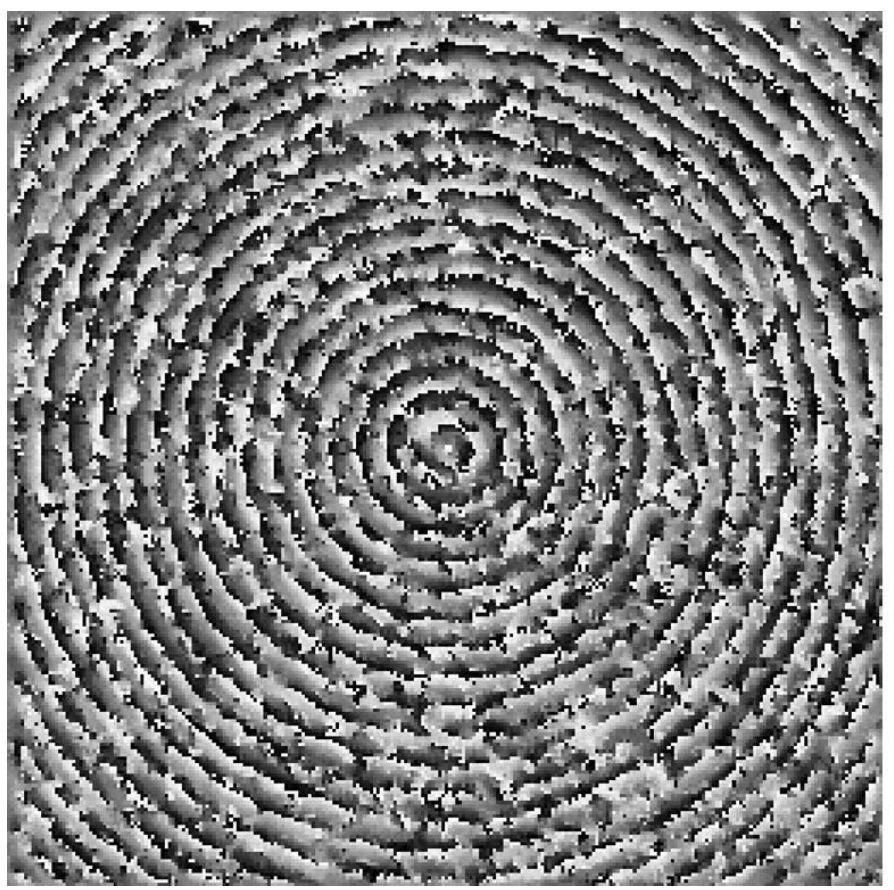

(c)

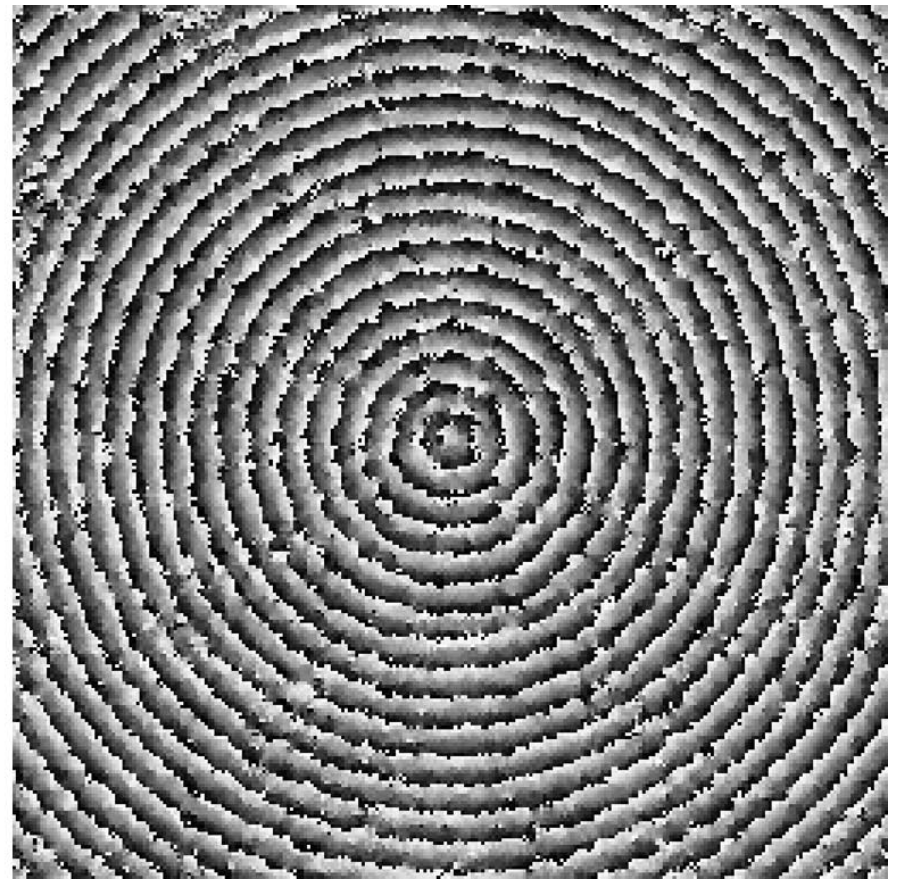

(b)

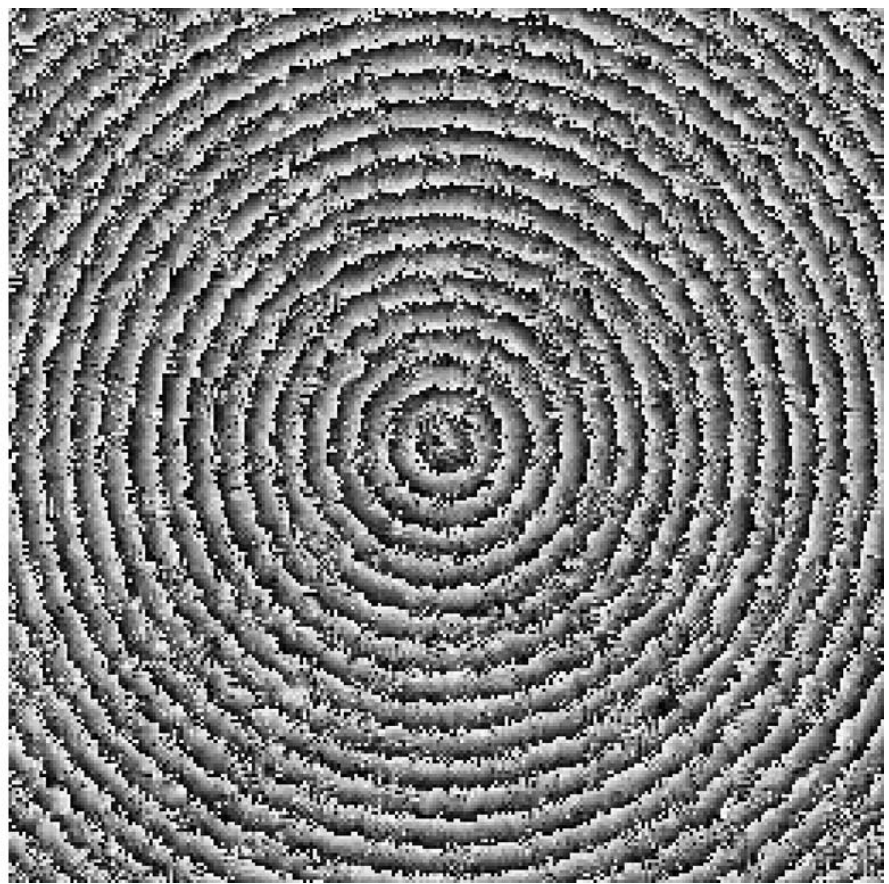

(d)

Fig. 9. Interferometric phase filtering results with an interferogram representing a cone with a six-pixel fringe period $|\rho|=0.4$. (a) Original noisy image. (b) WInPF filter. (c) Lee filter. (d) FFT-based filter.

decreases, and $\Gamma_{\text {sig }}$ decreases too. To detect the wavelet signal coefficients, a threshold is applied to $\Gamma_{\text {sig }}$

$$
\begin{aligned}
& \Gamma_{\text {sig }} \geq t h_{w} \Rightarrow \text { Signal coefficient } \\
& \Gamma_{\text {sig }}<t h_{w} \Rightarrow \text { Noise coefficient. }
\end{aligned}
$$

The threshold $t h_{w}$, which is equal for all the 16 signal bands, defines up to which coherence level the signal is processed. Normal values for $t h_{w}$ range from -1 to -5 . Lower values can be used to filter low-coherence areas $(|\rho| \lesssim 0.4)$. Finally, a mask indicating signal coefficients is generated using (37). In the mask, those isolated coefficients that are detected as signal coefficients are removed in order to reduce noise effects in the mask.

Step 3 (Signal Increase): The real and imaginary parts of those coefficients detected as signal in the previous step are multiplied by two. For the noise coefficients the real and imaginary parts are maintained.

Step 4 (Inverse DWT): The inverse DWT is applied but only reducing one wavelet scale. 


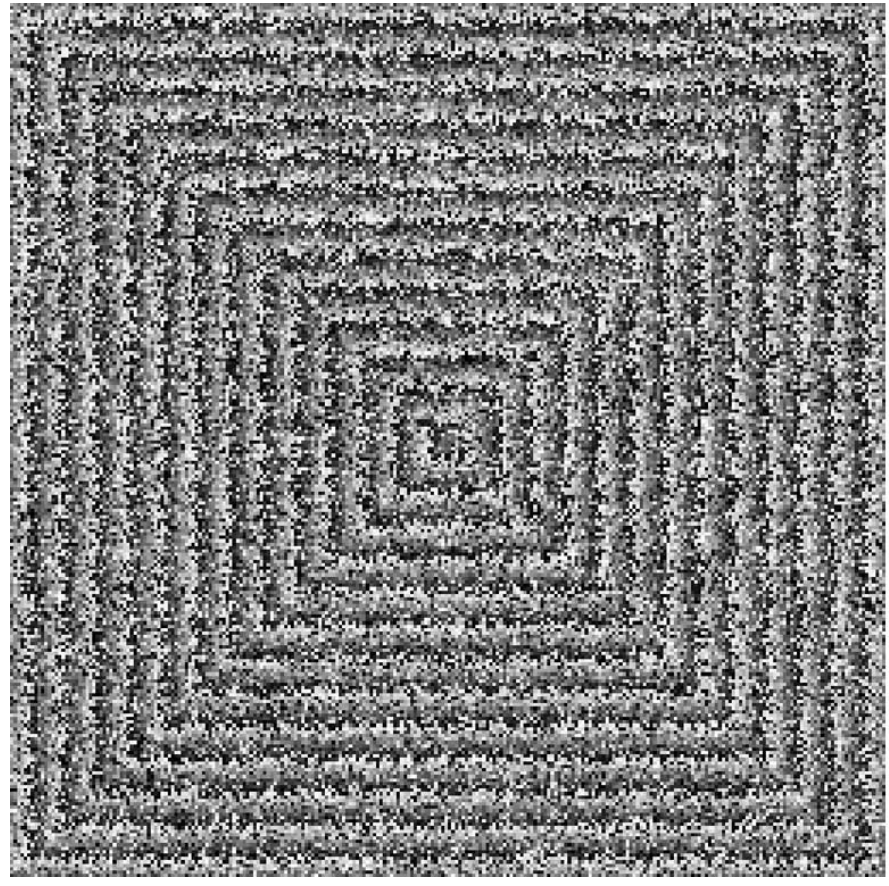

(a)

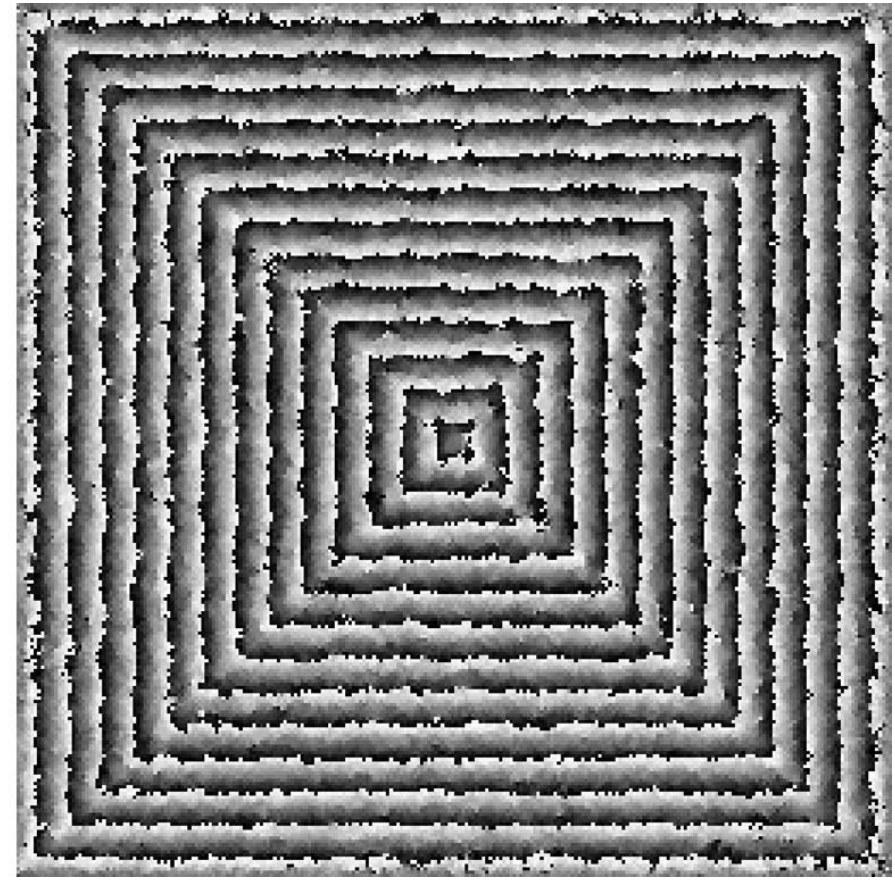

(b)

Fig. 10. Filtering result with the WInPF filter for an interferogram representing a pyramid with a ten-pixel fringe period $|\rho|=0.5$.

Step 5 (Signal Mask Growing): To obtain a mask locating signal coefficients for previous scales (higher frequency scales), a new mask is derived from the one generated in Step 2. Each four bands in a scale $2^{i}$ are derived from a single band in the previous wavelet scale $2^{i-1}$, where a 1-to-4 space relation is established between wavelet coefficients. First, the masks of the four bands at the scale $2^{i}$ are merged through a logical OR operation. Then, the dimensions of the merged mask are doubled to fit the $2^{i-1}$ scale band dimensions. In this case, if a pixel of the merged mask is classified as signal, the four pixels referring to the same spatial area, but in the band of the scale $2^{i-1}$, are also classified as signal; otherwise they are classified as noise. This sequence of mask growing allows to obtain a mask locating useful signals in the original domain.

The sequence of Steps 3-5 are repeated each time a wavelet scale is inversely transformed. As the wavelet transformation has been applied with three scales, this sequence has to be repeated three times. After the third iteration, a complex signal is obtained in the original domain.

Step 6(Phase Calculation): From the complex output of the algorithm, the phase is calculated, which is the estimation of the original interferometric phase.

\section{EXPERIMENTAL RESULTS}

In this section, the capabilities of the algorithm presented previously (in terms of noise reduction, spatial resolution maintenance, and computational efficiency) are presented. First, a quantitative comparison with alternative interferometric phase filters addressed in the literature is reported. Then, the performance of the new filter is tested, employing real interferograms taken with spaceborne as well as airborne SAR systems.

\section{A. Quantitative Analysis}

The capability of the proposed filter, called wavelet interferometric phase filter (WInPF) in the following, to reduce noise and to maintain spatial resolution, even on steep topographic slopes, is evaluated and compared with other filtering approaches. To perform such a quantitative comparison, simulated $256 \times 256$ pixel interferograms, representing a cone and a pyramid with one-look phase noise with coherences from 0.9 to 0.4 , have been employed.

The WInPF filter, calculating the DWT with a truncated Shannon filter and $t h_{w}=-1$, is compared with the complex multilook filter [10] (using a $5 \times 5$-pixel averaging window), a filter based on the fast Fourier transform (FFT) [12] (with a $6 \times 6$-pixel FFT window and an exponent of 1), and the filter presented in [9]. In order to compare the noise reduction properties, two mean-square errors (mse) have been used. The mse between the filtered and original interferometric phase without noise is calculated in the real and in the complex domain. In the first case, this mse mainly accounts for errors in $2 \pi$ phase jump areas, providing information about errors due to an erroneous filtering of such phase jumps. On the other hand, the mse is calculated in the complex domain, where phase jumps are not present. Table I shows the numerical comparison for a $256 \times 256$-pixel simulated interferogram, representing a cone, with a fringe period of six pixels. The WInPF filter has the lowest error over all the coherence values. It also presents the highest residues reduction, ranging from $100 \%$ in the case of $|\rho|=0.9$, to a reduction of $95 \%$ for $|\rho|=0.5$. Referring to the execution times, they have been obtained with a computer based on a $600-\mathrm{MHz}$ Intel Celeron processor equipped with $200 \mathrm{MB}$ random access memory. Due to the facts that the WInPF filter is not based on a pixel-by-pixel processing and 


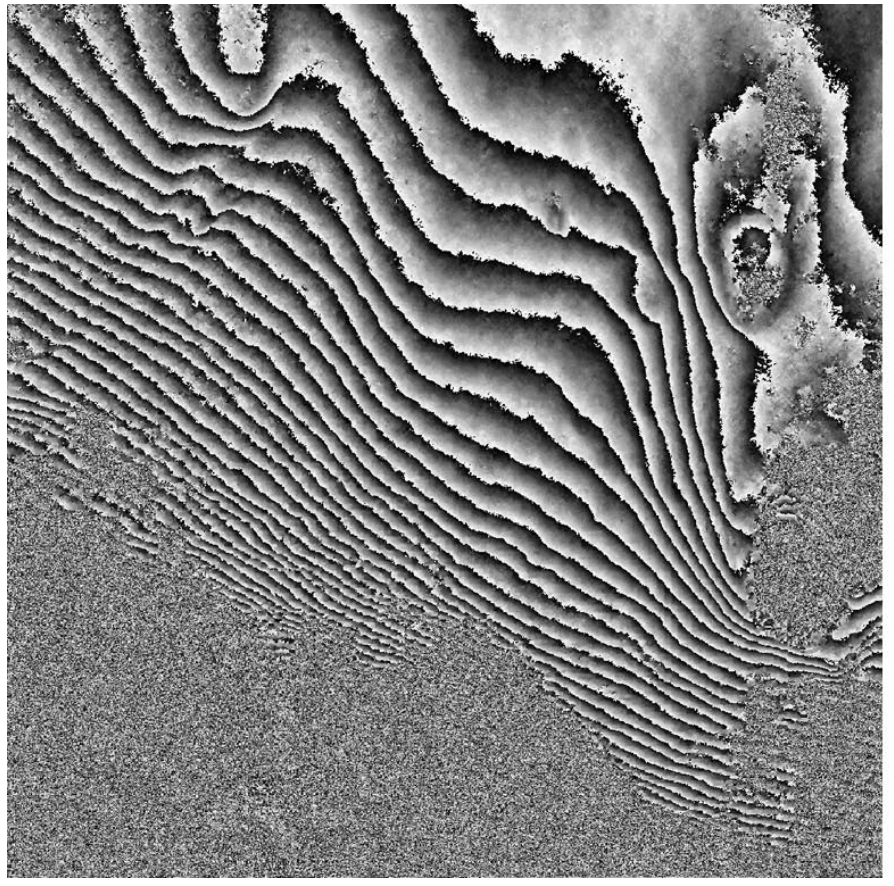

(a)

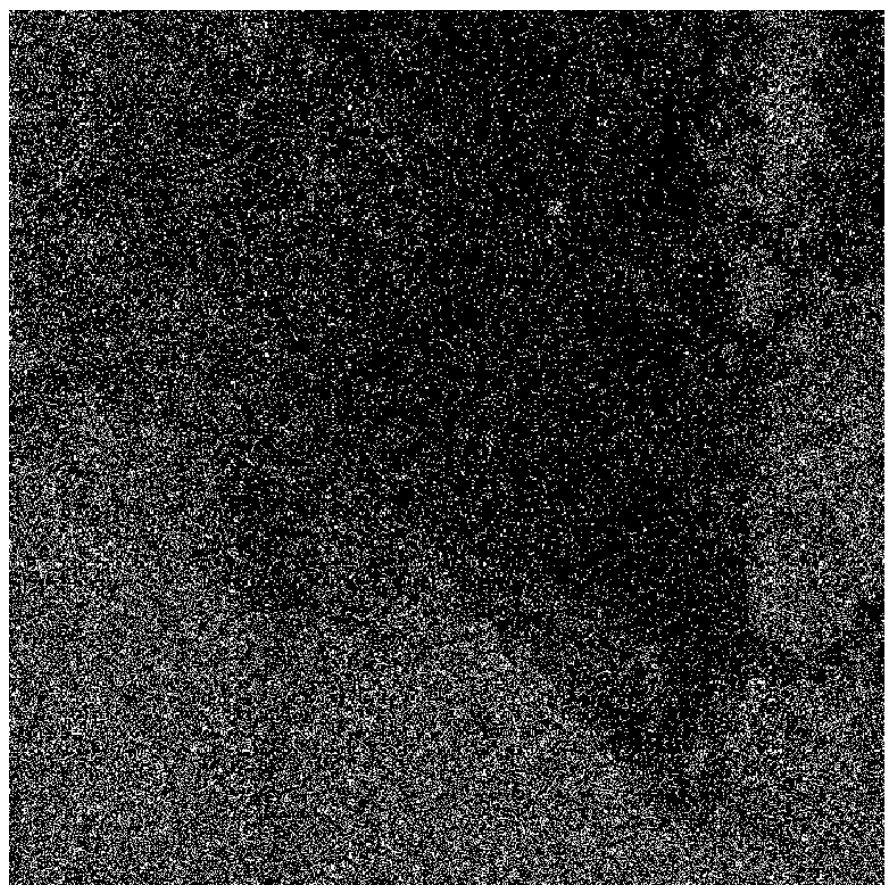

(c)

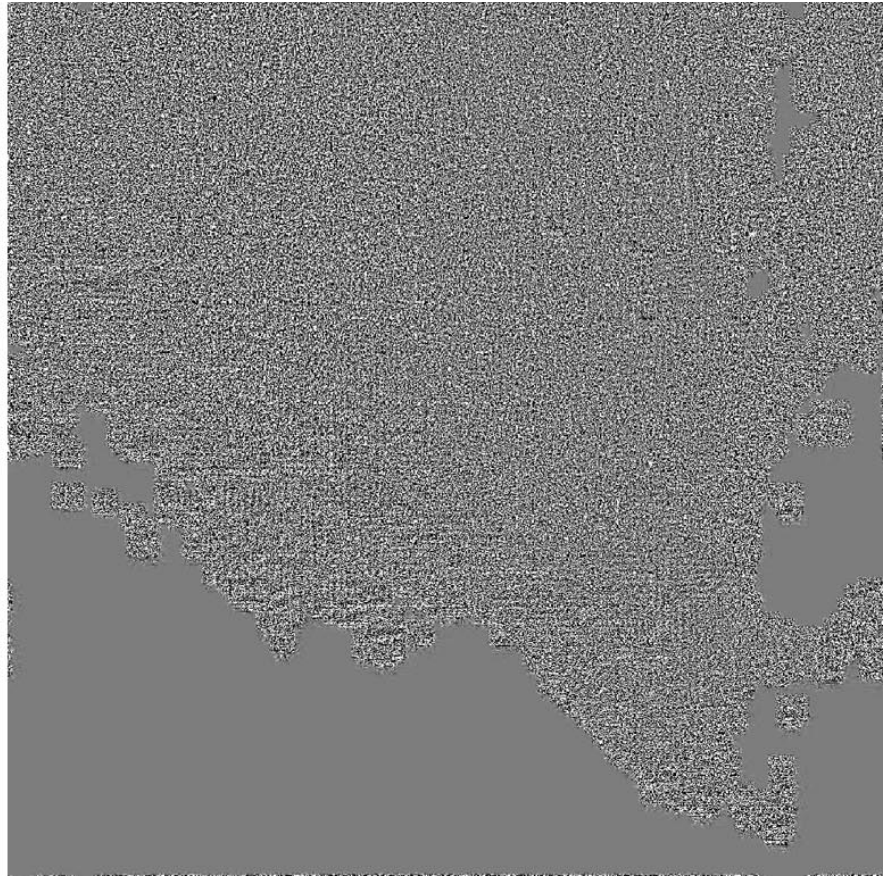

(b)

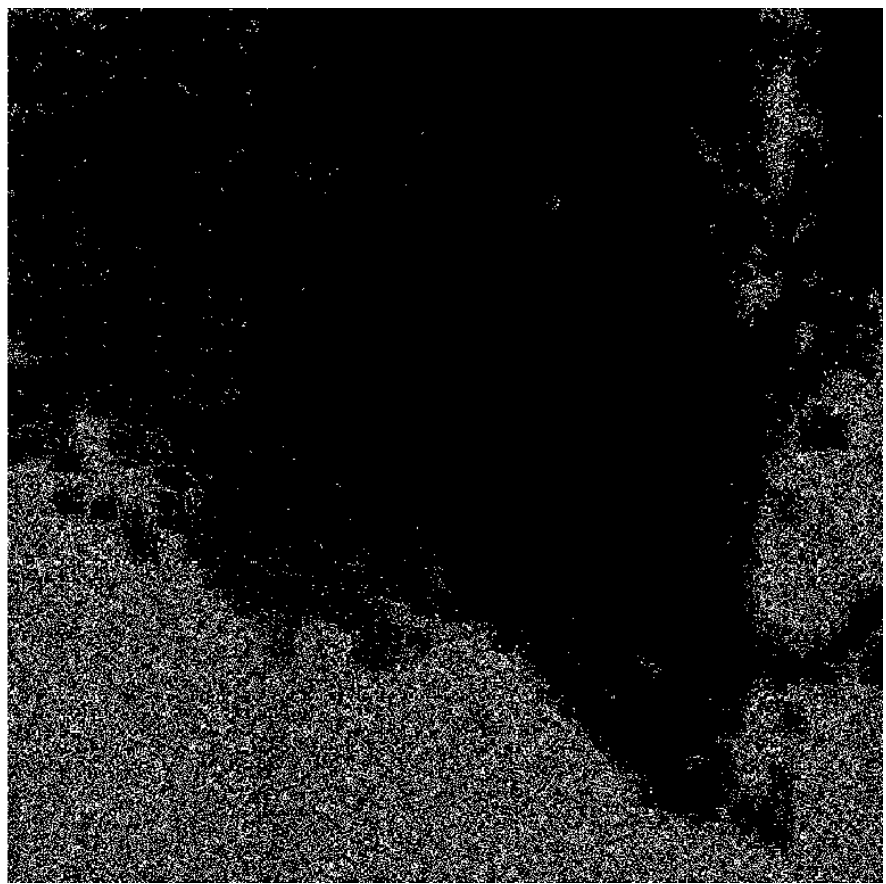

(d)

Fig. 11. Filtering result with the WInPF filter of the interferometric phase shown in Fig. 3(c). (a) Filtered interferometric phase. (b) Difference image. (c) Original phase residues map. (d) Filtered phase residues map.

that the DWT is calculated with a fast algorithm, low execution times, allowing to process large images, can be obtained.

Fig. 9 demonstrates the filter performance regarding the recovering of close fringes at low coherence values. In this case, the cone interferogram is employed, but with one-look phase noise of coherence equal to 0.4. From Fig. 9(b), it is possible to see that the proposed approach clearly reduces phase noise, recovering close fringes without introducing any kind of artifact within the filtered image. The use of a cone demonstrates that the new algorithm is able to process any fringe direction in the same efficient way. Fig. 9(c) and (d) presents respectively the filtering results using the filter presented in [9] and the FFT filter.

In order to test the spatial resolution maintenance properties of the WInPF filter, a $256 \times 256$-pixel interferogram representing a pyramid with phase noise coherence equal to 0.5 and a ten-pixel fringe period have been employed. The critical point is to maintain the pyramid edges. Fig. 10 shows the noisy phase image and the result obtained after applying the WInPF filter. The pyramid edges are clearly maintained. 


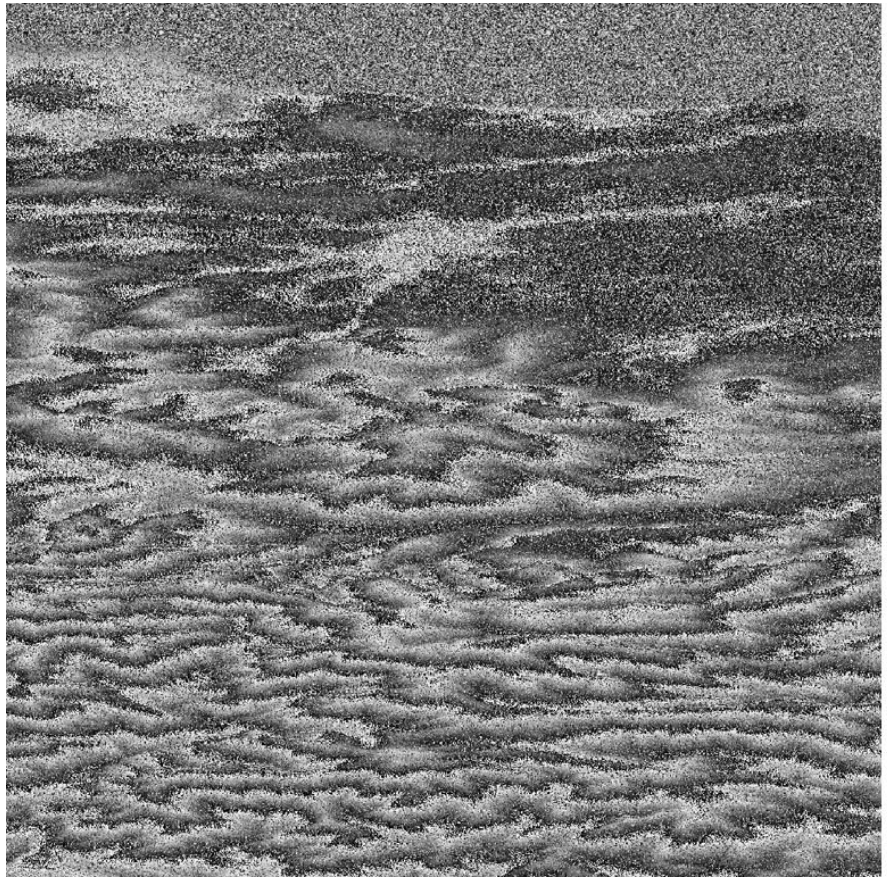

(a)

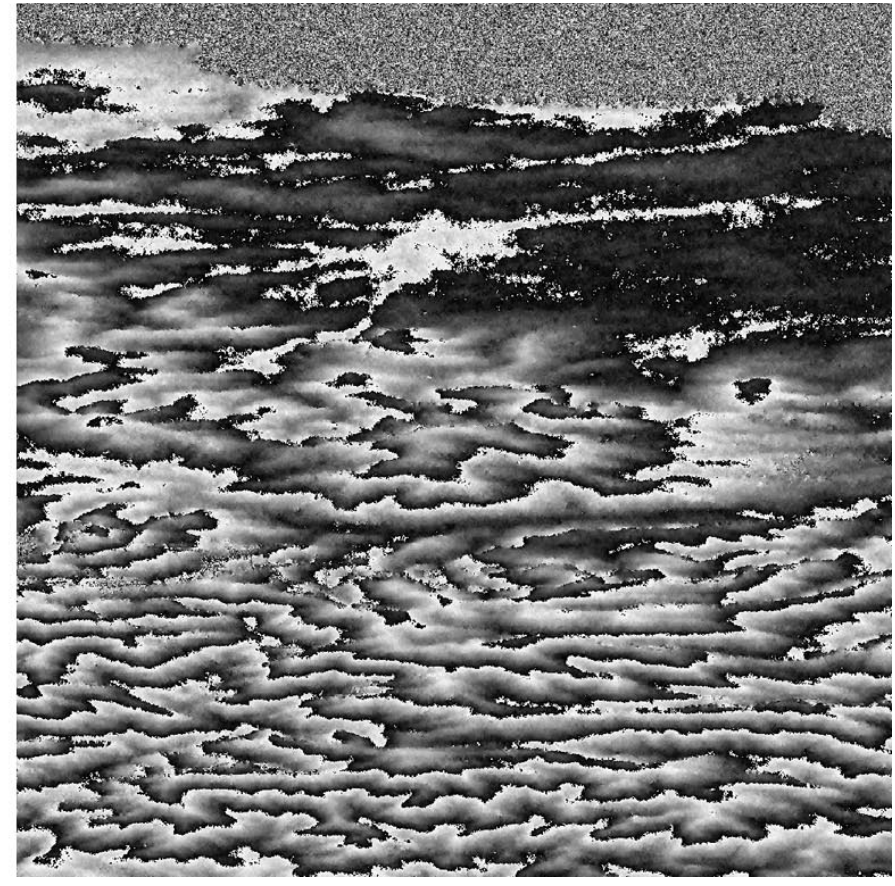

(b)

Fig. 12. Filtering result with the WInPF filter. (a) Original ERS-1 and ERS-2 interferometric phase over Tarragona, Spain. (b) Filtered interferometric phase.

\section{B. Real Interferograms Processing}

To demonstrate the filter's performance, also two different experimental interferograms, with different morphologies, have been filtered.

First, an E-SAR X-band interferometric phase of Mount Etna [already shown in Fig. 3(c)] has been filtered. This phase image has been filtered by the WInPF filter using, in this case, a Daubechies wavelet filter [14] of ten coefficients and $t h_{w}=-3$. From Fig. 11(a), one can notice that the algorithm is able to process, in the same efficient way, areas with smooth or steep slopes at the same time. Fig. 11(b) shows the difference between the original and the filtered phases, which has a mean equal to zero and does not contain any image detail, demonstrating that the proposed filter preserves the topographic information. As designed, the algorithm does not process very low coherence areas, avoiding to create false information. Phase residues reduction is about 50\%, but comparing Fig. 11(c) with Fig. 11(d), it becomes evident that they have almost been eliminated from those areas with useful signal.

As a second image, an ERS-1 and ERS-2 C-Band interferogram of Serra de Cardó in Tarragona, Spain has been processed. This one-look interferometric phase has also a 1024 $\times$ 1024-pixel dimension, but with an approximate spatial resolution of $30 \times 30 \mathrm{~m}$. Fig. 12(a), from the top to the bottom, shows the Mediterranean Sea with zero coherence, a flat area, and a mountainous region with a maximum height of $1200 \mathrm{~m}$, containing severe geometric distortions. Fig. 12(b) presents the filtered interferometric phase employing again a Daubechies wavelet filter of ten coefficients and $t h_{w}=-1$. Residues reduction is about $80 \%$ in the filtered image, which are concentrated on the sea and the areas with strong topography. From Fig. 12(b), it is noticeable how the proposed WInPF filter is able to process areas containing complicated topographic structures.

\section{Resolution Maintenance}

It is difficult to describe numerically resolution maintenance capabilities. Fig. 13(a) shows a detailed E-SAR X-band interferometric phase image of Oberpfaffenhofen, Germany, with 3 $\times 3-\mathrm{m}$ spatial resolution. This area is completely flat, so image details are mainly due to man-made targets-in this case, buildings. Fig. 13(b) presents the filtered image using the WInPF filter. Fig. 13(c) depicts the plot of a cut indicated in Fig. 13(b). From this graphic, it is possible to see how the algorithm is able to maintain phase jumps introduced by the buildings without damaging its shape. Not reducing the value of the wavelet coefficients allows not to lose any phase information maintaining high-frequency details.

\section{CONCLUSION}

In this paper, a noise model for the interferometric phase noise in the complex domain is introduced and validated using simulated and experimental interferometric phase images. Based on this model, also a novel noise model for the complex wavelet domain is derived. The availability of this model allows to implement a new filtering algorithm to reduce SAR interferometric phase noise in the complex wavelet plane. The filtering algorithm is not based on a windowing or segmenting process. On the contrary, the phase image is locally analyzed in the wavelet domain, and therefore, the filter is characterized by good spatial resolution maintenance properties and computational efficiency, as demonstrated. The algorithm allows also not to process those areas with very low coherence, avoiding the introduction of artifacts or false information. The algorithm's performance in terms of noise reduction and fringe preservation is analyzed and demonstrated with different 


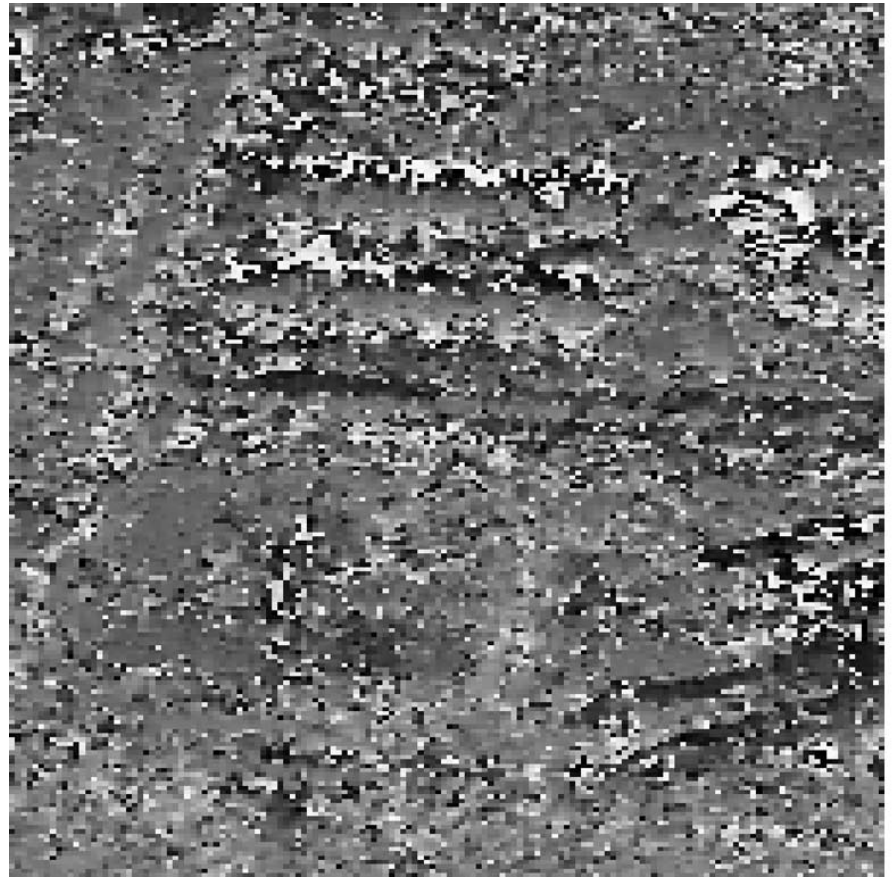

(a)

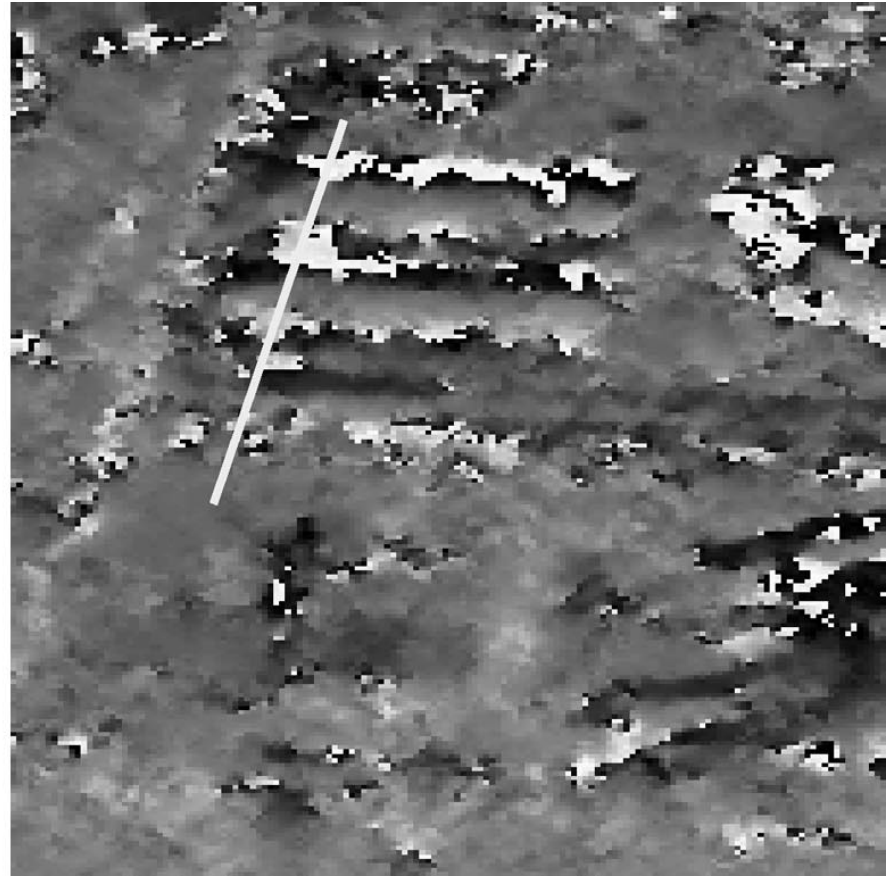

(b)

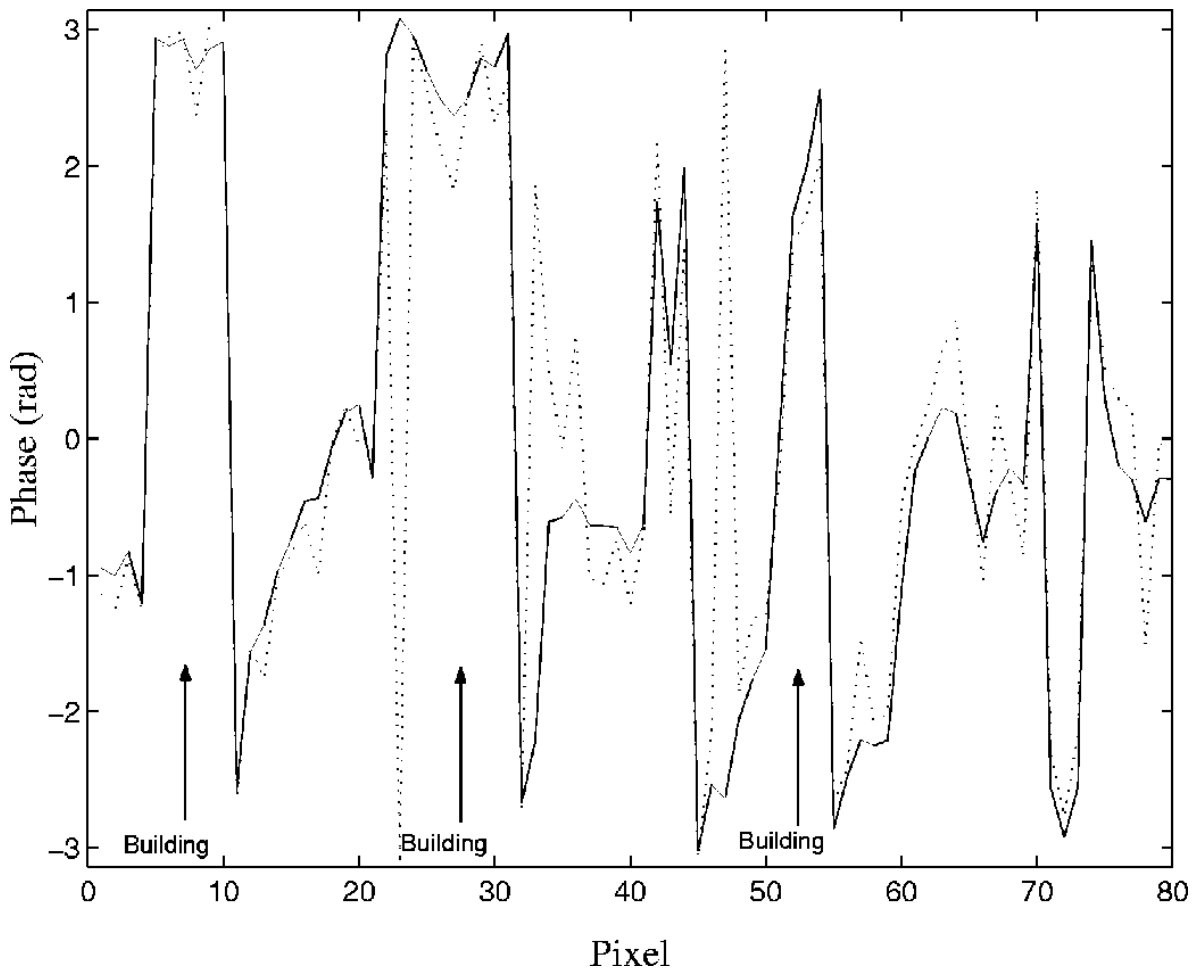

(c)

Fig. 13. Spatial resolution maintenance result. (a) Detailed image of the original interferometric phase. (b) Detailed image of the filtered phase. (c) Image cut of the white line shown in (b). Dashed line represents the original phase, and the solid line represents the filtered version.

interferometric phase images. A comparison with alternative approaches is reported.

\section{REFERENCES}

[1] R. Bamler and P. Hartl, "Synthetic aperture radar interferometry," Inv Prob., vol. 14, pp. R1-R54, 1998.
[2] L. C. Graham, "Synthetic interferometric radar for topographic mapping," Proc. IEEE, vol. 62, pp. 763-768, June 1974.

[3] R. Kwok and M. Fahnestock, "Ice sheet motion and topography from radar interferometry," IEEE Trans. Geosci. Remote Sensing, vol. 34, pp. 189-200, Jan. 1996.

[4] S. R. Cloude and K. P. Papathanassiou, "Polarimetric SAR interferometry," IEEE Trans. Geosci. Remote Sensing, vol. 36, pp. 1551-1565, Sept. 1998. 
[5] R. Goldstein, H. A. Zebker, and C. L. Werner, "Satellite radar interferometry: Two dimensional phase unwrapping," Radio Sci., vol. 23, pp. 713-720, 1988

[6] D. C. Ghiglia and M. D. Pritt, Two-Dimensional Phase Un-Wrapping: Theory, Algorithms, and Software. New York: Wiley, 1998.

[7] J. S. Lee, K. W. Hoppel, and S. A. Mango, "Intensity and phase statistics of multilook polarimetric and interferometric SAR imagery," IEEE Trans. Geosci Remote Sensing, vol. 32, pp. 1017-1027, Sept 1994.

[8] R. J. A. Tough, D. Blacknell, and S. Quegan, "A statistical description of polarimetric and interferometric synthetic aperture radar data," Proc. R. Soc. London A, pp. 567-589, 1995.

[9] J. S. Lee, K. P. Papathanassiou, T. L. Ainsworth, M. H. Grunes, and A. Reigber, "A new technique for noise filtering of SAR interferometric phase images," IEEE Trans. Geosci. Remote Sensing, vol. 36, pp. 1456-1465, Sept. 1998.

[10] M. S. Seymour and I. G. Cumming, "Maximum likelihood estimation for SAR interferometry," in Proc. IGARSS, Aug., 8-12 1994, pp. 2272-2275.

[11] J. S. Lee, "Digital image enhancement and noise filtering by use of local statistics," IEEE Trans. Pattern Anal. Machine Intell., vol. PAMI-2, pp. 165-168, Jan. 1980.

[12] H. Goldstein and C. Werner, "Radar ice motion interferometry," in Proce. 3rd ERS Symp., Florence, Italy, Mar. 1997, pp. 969-972.

[13] C. Oliver and S. Quegan, Understanding Synthetic Aperture Radar Images. Boston, MA: Artech House, 1998.

[14] S. Mallat, A Wavelet Tour of Signal Processing, 2nd ed. San Diego, CA: Academic, 1998

[15] A. Cohen and J. Kovacevic Jr., "Wavelets: The mathematical background," Proc. IEEE, vol. 84, pp. 514-522, Apr. 1996

[16] N. Hess-Nielsen and M. V. Wickerhauser, "Wavelets and time frequency analysis," Proc. IEEE, vol. 84, pp. 523-540, Apr. 1996.

[17] B. Vidakovic, Statistical Modeling by Wavelets. New York: Wiley, 1999.

[18] F. Gatelli, A. M. Guarneri, F. Parizzi, P. Pasquali, C. Prati, and F. Rocca, "The wavenumber shift in SAR interferometry," IEEE Trans. Geosci. Remote Sensing, vol. 32, pp. 855-865, July 1994.

[19] J. M. Shapiro, "Embedded image coding using zerotrees of wavelet coefficients," IEEE Trans. Signal Processing, vol. 41, pp. 3445-3462, Dec. 1993.

[20] D. E. Carrasco, "SAR interferometry for digital elevation model generation and differential applications," Ph.D. thesis, Univ. Politècnica de Catalunya, Barcelona, Spain, 1998.
[21] K. Ramchandran, M. Vetterli, and C. Herley, "Wavelets, sub-band coding and best bases," Proc. IEEE., vol. 84, pp. 541-558, Apr. 1996.

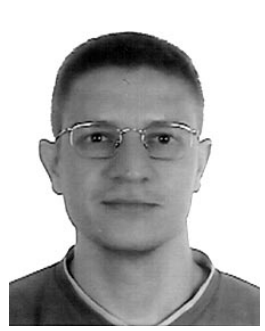

Carlos López-Martínez was born in Barcelona, Spain. He received the degree in electrical engineering from the Technical University of Catalonia (UPC), Barcelona, Spain, in 1998. He is currently pursuing the $\mathrm{Ph} . \mathrm{D}$. degree in electronic engineering at the Technical University of Catalonia.

In 1999, he joined the Signal Theory and Communications Department (TSC), Technical University of Catalonia. His Ph.D. thesis is focused on speckle noise modeling as well as in the definition of new algorithms for speckle reduction maintaining the spatial resolution. From October 2000 until March 2002, he joined the High Frequency and Radar Systems Department, German Aerospace Center (DLR), Oberpfaffenhofen, Germany. His research interests include SAR, SAR interferometry and polarimetry, digital image processing, and signal decomposition techniques.

Mr. López-Martínez received the Student Prize Paper Award in the EUSAR 2002 Conference, Cologne, Germany.

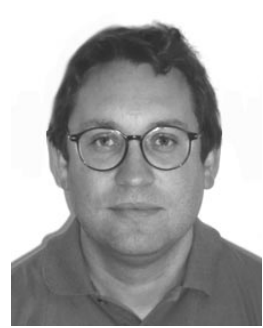

Xavier Fàbregas received the B.S. degree in physics from Barcelona University, Barcelona, Spain, in 1988, and the Ph.D. degree in applied sciences from the Technical University of Catalonia (UPC), Barcelona, Spain, in 1995.

Since 1996, he has been Associate Professor with UPC. In 2001, he spent an eight-month sabbatical period at the High Frequency and Radar Systems Department, German Aerospace Center (DLR), Oberpfaffenhofen, Germany. His current research interests include polarimetric retrieval algorithms, polarimetric calibration, polarimetric SAR classification, and polarimetric weather radar analysis. 